\title{
Statistical analysis of foliicolous fungal biodiversity of Konkan region, Maharashtra, India: A novel approach
}

\section{Dubey R and Pandey AD}

Botanical Survey of India, Western Regional Centre, Pune, Maharashtra, India

Dubey R, Pandey AD 2019 - Statistical analysis of foliicolous fungal biodiversity of Konkan region, Maharashtra, India: A novel approach. Plant Pathology \& Quarantine 9(1), 77-115, Doi $10.5943 / \mathrm{ppq} / 9 / 1 / 9$

\begin{abstract}
Foliicolous micro-fungi are the microscopic fungi inhabiting leaf surface as asymptomatic mutualists, benign commensals or latent pathogens. Maharashtra being the largest state in terms of area in peninsular India shows wide variety of ecosystems. With a view of studying the foliicolous fungi in such a diverse array of habitats, a project entitled "Foliicolous fungi of Maharashtra" was undertaken and various areas were visited in different forest ecosystems of Maharashtra. The present paper deals with the analysis of foliicolous micro-fungal diversity of Konkan region of Maharashtra. A total 191 foliicolous micro-fungi were found. In order to analyse difference in biodiversity across the districts ordinary least squares (OLS) and count data regression models were developed with Species Count as a dependent variable. Both approaches yielded identical results in terms of overall significance of regression and statistical significance of estimated coefficients: there was highly statistically significant $(p<0.01)$ difference in mean Species Count across the districts. To analyse the similarity in species composition Jaccard similarity Index (JSI) was calculated. Maximum JSI was $7.927 \%$, for Greater Thane and Sindhudurg districts. No species was common in all districts; only $4.396 \%$ species were common in maximum three districts (i.e. Greater Thane, Ratnagiri, Sindhudurg).
\end{abstract}

Key words - Foliicolous fungi - Jaccard similarity Index - Konkan - Mycobiota - Negative Binomial Regression Model (NBRM) - Ordinary Least Squares - Poisson Regression Model (PRM)

\section{Introduction}

Leaf surfaces continuously receive fungal spores deposited by wind impaction, sedimentation and rain wash. Though long considered as hostile environment for fungal growth, the leaf surface is a complex biological system, where interesting interactions occur between leaf and its intimately associated mycoflora (Andrews \& Harris 2000). Kinkel (1997) reported that a thin nutrient film deposited from the atmosphere on the leaf surface further facilitates the microbial colonization as growing plants make new surfaces available for fungi. They may act as asymptomatic mutualists, benign commensals or latent pathogens, depending upon the physico-chemical environment of leaf, any imbalance in which can change the nature of host-fungi relationship. Such imbalance often leads to the pathogenic phase of fungi manifesting in different kinds of symptoms (Andrews \& Hirano 1991). The potential of foliicolous fungi is not known much, from an application point of 
view; however, they constitute a fairly big group of micro-fungi.

Maharashtra is one of the larger states in India, with the coastline on its western side measuring about $825 \mathrm{~km}$ (Karve 1968). Konkan is a coastal narrow strip of Maharashtra, which starts from Tapti basin in North to Goa in South and lies to the east of Arabian Sea and the west of Sahyadris. Area of Konkan is $30,746 \mathrm{~km}^{2}$ and is 27 to $48 \mathrm{~km}$ broad (Singh \& Karthikeyan 2000). It comprises of seven districts of Maharashtra viz. Mumbai, Mumbai (Sub-urban), Thane, Palghar, Raigad, Ratnagiri and Sindhudurg. For the purpose of study, Greater Thane district has been defined to include districts of Mumbai, Mumbai (Sub-urban), Thane and Palghar. The reason being, Sanjay Gandhi National Park which is spread over the Mumbai (Sub-urban) and Thane districts, also because forest areas of Thane and Palghar districts are contiguous with each other. As far as forest types are concerned, Montane subtropical evergreen forests are found in Thane and Raigad districts. Subtropical evergreen forests are encountered in Amboli ghat (Savantwadi) and surroundings. West coast semi-evergreen forests are found in some parts of Ratnagiri and Sawantwadi.

Review of pertinent literature reveals that foliicolous fungi have been fairly well documented from some parts of India (Sahni 1964, Kumar \& Kamal 1979, Kamal \& Singh 1980, Rai \& Kamal 1982, Rajak \& Pandey 1984, Kamal \& Morgan-Jones 1985, Verma \& Kamal 1987a, 1987b, 1991, Bhat \& Kendrick 1993, Bhat \& Raghukumar 2000, D’Souza 2002, Bhat 2010, Pratibha et al. 2010, Hosagoudar \& Robin 2011, Mall et al. 2013, Thimmaiah et al. 2013). In Maharashtra, although serious efforts were not made to document the foliicolous fungi exclusively, but studies carried out earlier revealed the distinct presence of fungi on leaves (Parandekar 1964, Patwardhan 1969, Patil \& Magdum 1979, Pande \& Bansude 1980, Pande 1981, Patil \& Pawar 1989, Sawant \& Papdiwal 2007, Singh et al. 2011). Thus, it is evident from the above-cited literature that no systematic and serious studies of foliicolous fungi have been undertaken and only sporadic reports are available. To address these deficiencies, a project entitled "Foliicolous fungi of Maharashtra", was undertaken which involved a reconnaissance survey (2010-2015) in the natural stands and forest plantations throughout the Maharashtra State, encompassing different types of forest ecosystems. Enumeration of foliicolous fungi of Maharashtra along with associated symptoms has been recently been published by Dubey \& Pandey (2018). Floristic works generally tend to be enumerative. Present paper, however, focusses on foliicolous fungi of Konkan region of Maharashtra along with statistical analysis of the biodiversity to gain deeper understanding of underlying patterns of biodiversity.

\section{Methodology}

Foliicolous samples were collected from 37 locations in four districts (Fig. 1) - Greater Thane (10), Raigad (7), Ratnagiri (13) and Sindhudurg (7). The identification and description of fungi were made with the help of various books, monographs and review published in standard journals and books. Ainsworth (1973), von Arx (1981) offer keys to most of the groups of fungi. Fungi forming spores in Pcynidia or Acervuli were identified from Nag Raj (1993), Sutton (1980). Subramanian (1971), Barnett \& Hunter (1972), Ellis (1971, 1976), Ellis \& Ellis (1985). Seifert (2011) was referred for the Hypomycetes. Ascomycetes were identified from Dennis (1978). Alexopolous \& Mims (1979) was also consulted. A manual by Cummins \& Hirsukta (1983) was referred for the identification of various rust and smut species. Bitunicate ascomycetes were studied with the help of Sivanesan (1983). Booth (1971), Smith \& Onions (1994), Butler \& Bisby (1931), Mundkar (1938) were very useful in identification of pathogenic groups. Hosagoudar (1996, 2008, 2012, 2013) were consulted for the identification of Black mildew fungi. Total number of fungi was 191, which have been enumerated in Table 1 along with associated host and location data. The district locations along with fungal species, observed are given in supplementary material. 


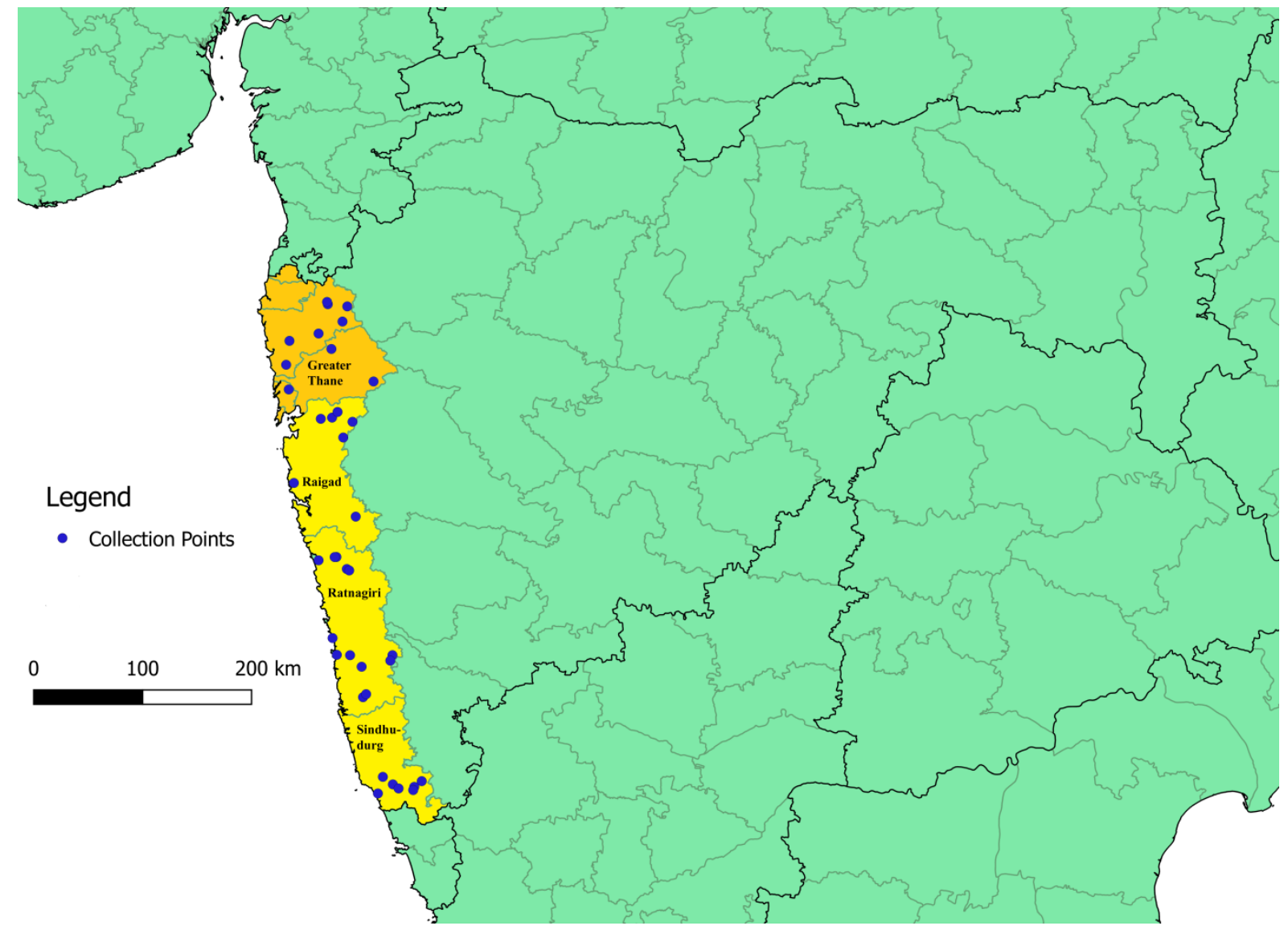

Fig. 1 - Collection locations in different districts of Konkan region (Greater Thane highlighted in orange; Raigad, Ratnagiri and Sindhudurg, in yellow) of Maharashtra, India

\section{Statistical Methods}

In order to study the difference in biodiversity across the four districts, additive dummy variable regression model of following functional form was developed:

$$
\text { SpsCount }=\beta_{0}+\sum_{j=1}^{n} \beta_{j} D_{j}+\epsilon
$$

Where $D_{j}$ is $j^{\text {th }}$ district dummy, $\beta_{\mathrm{j}}$ is its estimated $\mathrm{j}^{\text {th }}$ coefficient and $\beta_{0}$ is intercept, representing benchmark category against which comparisons are made. $\beta_{\mathrm{j}}$ is the difference in mean SpsCount between $\mathrm{j}^{\text {th }}$ category and benchmark category. SpsCount is number of species at each location. It is to be noted that a species can be found at more than one location, however at any given location a species has been counted only once. The reason for choosing SpsCount as a dependent variable is that it is a simplest measure of biodiversity, determined by whole range of biotic and abiotic factors. For the purpose of study, following functional form was considered:

$$
\text { Spscount }=\beta_{0}+\beta_{1} \mathrm{DS}+\beta_{2} \mathrm{DRd}+\beta_{3} \mathrm{DT}
$$

Where DS is Sindhudurg district dummy, DRd is Raigad district dummy, DT is Greater Thane dummy. Intercept term $\beta_{0}$ represents mean SpsCount for Ratnagiri district, chosen as benchmark category due to its lowest mean SpsCount.

Two classes of models were developed and their comparative performance carried out. First being, Classical Normal Linear Regression Model (CNLRM) estimated by Ordinary Least Squares 
(OLS). In order to develop CNLRM model, SpsCount is assumed to be continuous variable, which is rounded to nearest integer when interpreting regression output. Second being, count data models, estimated by Maximum Likelihood Estimation (MLE). Count data models have been developed as SpsCount is a count variable, taking only non-negative integer values. Count data models developed were Poisson Regression Model (PRM) and Negative Binomial Regression model (NBRM). For NBRM, Negbin2 form was chosen. This was developed to account for overdispersion, if present in PRM.

In OLS, F-test tests the null hypothesis that all the estimated slope coefficients are simultaneously equal to zero i.e. there is no difference in mean SpsCount across the districts. Thus, rejecting null hypothesis would mean that there is difference in mean SpsCount across the districts, implying difference infungal biodiversity across the districts of Konkan. MLE equivalent of F-test (in OLS) is Likelihood Ratio Statistic $(\lambda)$ which follows Chi-square distribution with (k-1) degrees of freedom, where $\mathrm{k}$ is number of regressors.

Regression diagnostics were carried out to ensure that the model assumptions are not violated so as to avoid drawing misleading conclusions. Details of models and associated regression diagnostics have been discussed in references (Greene 2011, Gujarati 2014). CNLRM model was tested for normality and homoskedasticity of residuals. Spatial autocorrelation was assumed away as model doesn't involve number of individuals clumping together. Breusch-Pagan test and White's test were used to test the null hypothesis of homoskedasticity of residuals of OLS models. Chisquare test was used to test the null hypothesis of normality of residuals of OLS as well as count data models. When residuals are not normally distributed, t-,z-,f- \& Chi-square- tests become suspect as they all are based on normality assumption. Heteroskedasticity in CNLRM and overdispersion in PRM lead to overestimated statistical significance of estimated coefficients. Gretl (Cottrell \& Lucchetti 2018) and Statsmodels (Seabold \& Perktold 2010) module for Python were used for regression analysis.

To analyse similarity in species composition between any two districts (A \& B), Jaccard Similarity Index (JSI) was used, given by:

$$
\text { JSI (between A \& B })=\frac{n(A \cap B)}{n(A \cup B)}=\frac{n(A \cap B)}{n(A)+n(B)-n(A \cap B)}
$$

\section{Results}

OLS models (Table 2$)$ showed highly statistically significant $\left(\mathrm{F}_{\mathrm{p} \text {-value }}<0.01\right)$ difference in mean SpsCount across the districts, with highly significant coefficients ( $\mathrm{p}$-value $<0.01$ ) for Sindhudurg and Greater Thane dummies, implying highly statistically significant difference in biodiversity across the districts was. However, when regression diagnostics was carried out, in Model 1 heteroskedasticity was highly significant ( $\mathrm{p}$-value $=0.0001$ for Breusch-Pagan Test, $\mathrm{p}$ value $=0.0089$ for White's Test) for both the tests. Also, in Model 1 residuals were not normally distributed ( $\mathrm{p}$-value $=0.023$ for Chi-square test). To overcome these limitations, Model 2 was developed, with natural log of SpsCount as dependent variable. This yielded identical results, with normally distributed residuals ( $\mathrm{p}$-value $=0.3228)$ and no evidence of heteroskedasticity $(\mathrm{p}$-value $=$ 0.7360 for Breusch-Pagan Test, $\mathrm{p}$-value $=0.6602$ for White's Test). Similar results were obtained for count data models (Table 3). PRM model showed statistically significant over dispersion (pvalue $=0.0223$ ), making results suspect. Hence, NBRM model (Negbin2) was developed, which yielded identical results, with statistically significant ( $\mathrm{p}$-value $=0.0913) \phi$, which is over dispersion parameter of NBRM. LLR p-value for both models ( $3 \& 4$ ) was less than $1 \%$, having highly significant coefficients ( $\mathrm{p}$-value $<0.01$ ) for Sindhudurg and Greater Thane dummies, with implications similar to F-test and t-test of OLS models. But, since error terms are not normally distributed for both count data models ( $\mathrm{p}$-value $=0.0229)$, as in case of OLS models, various tests of significance become suspect. However, by dropping observation for Kesari (SpsCount $=33$ ), residuals had normal distribution ( $\mathrm{p}$-value $=0.4997$ for modified Model 1, 3 and 4) with overall conclusion remaining the same at the same level of statistical significance $(\alpha=0.01)$, though 
Model 1 still showed similar heteroskedasticity (p-value $<0.01$ for both tests). Hence, the models with all the observations have been retained. All the four models show similar results at $\alpha=0.01$. Of all the 4 models developed, Model 1 is simplest in terms of functional form and ease of interpretability. For these reasons, Model 1was retained (though Model 2 has lowest AIC) and it was concluded that there is highly statistically significant $\left(\mathrm{F}_{\mathrm{p} \text {-value }}<0.01\right)$ difference in mean SpsCount - and thus biodiversity - across the districts of Konkan.

For studying similarity in species composition between any two districts, Jaccard Similarity Index (JSI) was calculated (Table 4). Maximum JSI was 7.927\%, between Greater Thane and Sindhudurg districts. None of the species were common in all the districts. Only 4 species (4.396\% of total) were common in maximum of 3 districts viz., Greater Thane, Ratnagiri, Sindhudurg. Thus, Konkan region shows highly rich biodiversity of foliicollous micro-fungi.

Table 1 Enumeration of fungal species along with associated host and location data.

\begin{tabular}{|c|c|c|c|c|c|c|c|c|}
\hline $\begin{array}{l}\text { Sr. } \\
\text { No. }\end{array}$ & Fungi & Host plants & $\begin{array}{l}\text { Collection } \\
\text { No. BSI } \\
(\text { WC) } \\
\end{array}$ & $\begin{array}{l}\text { Date of } \\
\text { collection }\end{array}$ & Sampling sites & $\begin{array}{l}\text { Latitude } \\
\text { (N) }\end{array}$ & $\begin{array}{l}\text { Longitude } \\
\text { (E) }\end{array}$ & $\begin{array}{l}\text { Altitude } \\
\text { (m) }\end{array}$ \\
\hline 1 & Acarophialophora sp. & Dillenia pentagyna Roxb. & 200145 & 21.01.2012 & $\begin{array}{l}\text { Fanaswadi, } \\
\text { Sindhudurg } \\
\text { Dist. }\end{array}$ & 15.890990 & 73.933043 & 151 \\
\hline 2 & $\begin{array}{l}\text { Acremoniula sarcinellae (Pat. \& } \\
\text { Har.) G. Arnaud ex Deighton }\end{array}$ & Apocynaceae sp. 1 & 201021 & 16.10.2012 & $\begin{array}{l}\text { On the way to } \\
\text { Zap, Greater } \\
\text { Thane }\end{array}$ & 19.898442 & 73.228724 & 375 \\
\hline 3 & $\begin{array}{l}\text { Acroconidiellina arecae (Berk. \& } \\
\text { Broome) M.B. Ellis }\end{array}$ & Areca catechu L. & 200061 & 20.01.2012 & $\begin{array}{l}\text { Sawantwadi, } \\
\text { Sindhudurg } \\
\text { Dist. }\end{array}$ & 15.904231 & 73.812647 & 184 \\
\hline 4 & $\begin{array}{l}\text { Acrodictys balladynae (Hansf.) } \\
\text { M.B. Ellis }\end{array}$ & $\begin{array}{l}\text { Catunaregam spinosa } \\
\text { (Thunb.) Tirveng. }\end{array}$ & 200993 & 24.01 .2013 & $\begin{array}{l}\text { Kodawali- } \\
\text { Rajapura, } \\
\text { Ratnagiri }\end{array}$ & 16.682105 & 73.544952 & 167 \\
\hline 5 & $\begin{array}{l}\text { Alternaria chlamydospora } \\
\text { Mouch. }\end{array}$ & Ficus religiosa L. & 196370 & 24.09 .2013 & $\begin{array}{l}\text { On the way to } \\
\text { Sanjay Gandhi } \\
\text { National Park, } \\
\text { Greater Thane }\end{array}$ & 19.398960 & 72.885344 & 71 \\
\hline 6 & $\begin{array}{l}\text { Amazonia elaeocarpi Hosag., } \\
\text { D.K. Agarwal, H. Biju \& } \\
\text { Archana }\end{array}$ & $\begin{array}{l}\text { Leea indica (Burm. f.) } \\
\text { Merr. }\end{array}$ & 200117 & 21.01.2012 & $\begin{array}{l}\text { Kesari, } \\
\text { Sindhudurg } \\
\text { Dist. }\end{array}$ & 15.917294 & 73.941860 & 165 \\
\hline 7 & $\begin{array}{l}\text { Amerosporium polynematoides } \\
\text { Speg. }\end{array}$ & $\begin{array}{l}\text { Eleusine coracana }(\mathrm{L} .) \\
\text { Gaertn. }\end{array}$ & 201123 & 18.10 .2012 & $\begin{array}{l}\text { Pulachiwadi, } \\
\text { Greater Thane }\end{array}$ & 19.880359 & 73.389547 & 395 \\
\hline
\end{tabular}


Table 1 Continued.

\begin{tabular}{|c|c|c|c|c|c|c|c|c|}
\hline $\begin{array}{l}\text { Sr. } \\
\text { No. }\end{array}$ & Fungi & Host plants & $\begin{array}{l}\text { Collection } \\
\text { No. BSI } \\
\text { (WC) }\end{array}$ & $\begin{array}{l}\text { Date of } \\
\text { collection }\end{array}$ & Sampling sites & $\begin{array}{l}\text { Latitude } \\
\text { (N) }\end{array}$ & $\begin{array}{l}\text { Longitude } \\
\text { (E) }\end{array}$ & $\begin{array}{l}\text { Altitude } \\
\text { (m) }\end{array}$ \\
\hline \multirow[t]{3}{*}{8} & \multirow[t]{3}{*}{ Ampelomyces quisqualis Ces. } & Malachra capitata L. & 201052 & 16.10 .2012 & $\begin{array}{l}\text { Jawhar, Greater } \\
\text { Thane }\end{array}$ & 19.917037 & 73.222610 & 433 \\
\hline & & $\begin{array}{l}\text { Abelmoschus esculentus } \\
\text { (L.) Moench }\end{array}$ & 201114 & 18.10 .2012 & $\begin{array}{l}\text { Pulachiwadi, } \\
\text { Greater Thane }\end{array}$ & 19.880359 & 73.389547 & 395 \\
\hline & & Malachra capitata $\mathrm{L}$. & 201161 & 19.10.2012 & $\begin{array}{l}\text { Tansa WLS, } \\
\text { Greater Thane }\end{array}$ & 19.529328 & 73.258630 & 295 \\
\hline 9 & Aphanofalx sp. & Bambusa bambos (L.) Voss & 200015 & 20.01 .2012 & $\begin{array}{l}\text { Akeri, } \\
\text { Sawantwadi } \\
\text { Sindhudurg } \\
\text { Dist. }\end{array}$ & 15.937989 & 73.765437 & 66 \\
\hline \multirow{2}{*}{10} & \multirow{2}{*}{$\begin{array}{l}\text { Ardhachandra cristaspora } \\
\text { (Matsush.) Subram. \& Sudha }\end{array}$} & $\begin{array}{l}\text { Jasminum odoratum } \\
\text { Noronha }\end{array}$ & 196359 & 24.09 .2013 & $\begin{array}{l}\text { Karjat, Raigad } \\
\text { Dist. }\end{array}$ & 18.928594 & 73.432723 & 122 \\
\hline & & $\begin{array}{l}\text { Flacourtia indica (Burm.f.) } \\
\text { Merr. }\end{array}$ & 196354 & 23.09 .2013 & \begin{tabular}{|l} 
Matheran, \\
Raigad Dist. \\
\end{tabular} & 18.963105 & 73.264070 & 742 \\
\hline \multirow[t]{4}{*}{11} & \multirow[t]{4}{*}{ Aschersonia aleyrodis Webber } & Helicteres isora $\mathrm{L}$. & 200206 & 22.01 .2012 & $\begin{array}{l}\text { Amboli Ghat, } \\
\text { Sindhudurg } \\
\text { Dist. }\end{array}$ & 15.964716 & 74.003571 & 700 \\
\hline & & Grewia sp. & 200230 & 22.01 .2012 & $\begin{array}{l}\text { Amboli Ghat, } \\
\text { Sindhudurg } \\
\text { Dist. }\end{array}$ & 15.964716 & 74.003571 & 700 \\
\hline & & Ziziphus jujuba Mill. & 200139 & 21.01.2012 & $\begin{array}{l}\text { Fanaswadi, } \\
\text { Sindhudurg } \\
\text { Dist. } \\
\end{array}$ & 15.890990 & 73.933043 & 151 \\
\hline & & Unidentified plant sp. 1 & 200162 & 21.01.2012 & $\begin{array}{l}\text { Kesari, } \\
\text { Sindhudurg } \\
\text { Dist. }\end{array}$ & 15.917294 & 73.941860 & 165 \\
\hline 12 & $\begin{array}{l}\text { Asperisporium pongamiae } \\
\text { (H.Syd.) Deighton }\end{array}$ & $\begin{array}{l}\text { Pongamia pinnata }(\mathrm{L} .) \\
\text { Pierre }\end{array}$ & 200033 & 16.10 .2013 & $\begin{array}{l}\text { Akeri, } \\
\text { Sawantwadi, } \\
\text { Sindhudurg } \\
\text { Dist. }\end{array}$ & 15.937989 & 73.765437 & 66 \\
\hline
\end{tabular}


Table 1 Continued.

\begin{tabular}{|c|c|c|c|c|c|c|c|c|}
\hline $\begin{array}{l}\text { Sr. } \\
\text { No. }\end{array}$ & Fungi & Host plants & $\begin{array}{l}\text { Collection } \\
\text { No. BSI } \\
\text { (WC) }\end{array}$ & $\begin{array}{l}\text { Date of } \\
\text { collection }\end{array}$ & Sampling sites & $\begin{array}{l}\text { Latitude } \\
\text { (N) }\end{array}$ & $\begin{array}{l}\text { Longitude } \\
\text { (E) }\end{array}$ & $\begin{array}{l}\text { Altitude } \\
\text { (m) }\end{array}$ \\
\hline & & $\begin{array}{l}\text { Pongamia pinnata }(\mathrm{L} .) \\
\text { Pierre }\end{array}$ & 201047 & 16.10 .2012 & $\begin{array}{l}\text { Jawhar, Greater } \\
\text { Thane }\end{array}$ & 19.917037 & 73.222610 & 433 \\
\hline 13 & $\begin{array}{l}\text { Asteridiella depokensis (Hansf.) } \\
\text { Hansf. }\end{array}$ & Vitex negundo $\mathrm{L}$. & 200081 & 21.01 .2012 & $\begin{array}{l}\text { Kudal, } \\
\text { Sindhudurg } \\
\text { Dist. } \\
\end{array}$ & 15.999956 & 73.683052 & 35 \\
\hline 14 & $\begin{array}{l}\text { Asterina capparis Syd., P. Syd. \& } \\
\text { E.J. Butler }\end{array}$ & Capparis sp. & 200224 & 22.01 .2012 & $\begin{array}{l}\text { Amboli Ghat, } \\
\text { Sindhudurg } \\
\text { Dist. }\end{array}$ & 15.964716 & 74.003571 & 700 \\
\hline 15 & $\begin{array}{l}\text { Asterina delicatula Syd., P. Syd. } \\
\& \text { Bal }\end{array}$ & Jasminum sp. & 200075 & 20.01 .2012 & $\begin{array}{l}\text { Sawantwadi, } \\
\text { Sindhudurg } \\
\text { Dist. }\end{array}$ & 15.904231 & 73.812647 & 184 \\
\hline 16 & $\begin{array}{l}\text { Asterina jambolanae A.K. Kar \& } \\
\text { Maity }\end{array}$ & Eugenia sp. & 200100 & 21.01 .2012 & $\begin{array}{l}\text { Kesari, } \\
\text { Sindhudurg } \\
\text { Dist. } \\
\end{array}$ & 15.917294 & 73.941860 & 165 \\
\hline \multirow[t]{2}{*}{17} & \multirow[t]{2}{*}{ Asterina jasminicola $\mathrm{W}$. Yamam } & Jasminum sp. & 200005 & 20.01.2012 & $\begin{array}{l}\text { Akeri, } \\
\text { Sawantwadi, } \\
\text { Sindhudurg } \\
\text { Dist. }\end{array}$ & 15.937989 & 73.765437 & 66 \\
\hline & & $\begin{array}{l}\text { Jasminum multiflorum } \\
\text { (Burm. f.) Andrews }\end{array}$ & 200080 & 21.01 .2012 & $\begin{array}{l}\text { Kudal, } \\
\text { Sindhudurg } \\
\text { Dist. } \\
\end{array}$ & 15.999956 & 73.683052 & 35 \\
\hline 18 & Asterina woodfordiae V.P. Sahni & $\begin{array}{l}\text { Woodfordia fruticosa }(\mathrm{L} .) \\
\text { Kurz. }\end{array}$ & 201106 & 17.10.2012 & $\begin{array}{l}\text { On the way to } \\
\text { Dahanu, Greater } \\
\text { Thane }\end{array}$ & 19.596222 & 72.912617 & 92 \\
\hline 19 & $\begin{array}{l}\text { Asterina wrightii Berk. \& M.A. } \\
\text { Curtis }\end{array}$ & $\begin{array}{l}\text { Paramignya monophylla } \mathrm{W} \\
\text { ight }\end{array}$ & 194051 & 26.01 .2013 & $\begin{array}{l}\text { Dapoli Forest } \\
\text { Range, Ratnagiri } \\
\text { Dist. }\end{array}$ & 17.786041 & 73.152662 & 60 \\
\hline 20 & Bahusandhika indica Subram. & Cocos nucifera L. & 194261 & 26.01 .2013 & \begin{tabular}{|l|} 
Dapoli, \\
Ratnagiri Dist.
\end{tabular} & 17.813476 & 73.300173 & 135 \\
\hline
\end{tabular}


Table 1 Continued.

\begin{tabular}{|c|c|c|c|c|c|c|c|c|}
\hline $\begin{array}{l}\text { Sr. } \\
\text { No. }\end{array}$ & Fungi & Host plants & $\begin{array}{l}\text { Collection } \\
\text { No. BSI } \\
\text { (WC) }\end{array}$ & $\begin{array}{l}\text { Date of } \\
\text { collection }\end{array}$ & Sampling sites & $\begin{array}{l}\text { Latitude } \\
\text { (N) }\end{array}$ & $\begin{array}{l}\text { Longitude } \\
\text { (E) }\end{array}$ & $\begin{array}{l}\text { Altitude } \\
\text { (m) }\end{array}$ \\
\hline 21 & Balladyna pavattae Boedijin & $\begin{array}{l}\text { Synedrella nodiflora (L.) } \\
\text { Gaertn. }\end{array}$ & 200026 & 20.01.2012 & $\begin{array}{l}\text { Akeri, } \\
\text { Sawantwadi, } \\
\text { Sindhudurg } \\
\text { Dist. }\end{array}$ & 15.937989 & 73.765437 & 66 \\
\hline 22 & $\begin{array}{l}\text { Balladyna vanderystii (Hansf.) } \\
\text { Arx }\end{array}$ & $\begin{array}{l}\text { Catunaregum spinosa } \\
\text { (Thunb.) Tirveng. }\end{array}$ & 200993 & 29.09 .2011 & $\begin{array}{l}\text { Kodawali- } \\
\text { Rajapura, } \\
\text { Ratnagiri }\end{array}$ & 16.682105 & 73.544952 & 167 \\
\hline 23 & Beltrania querna Harkn. & Eucalyptus sp. & 194127 & 16.10.2012 & $\begin{array}{l}\text { Wada,Greater } \\
\text { Thane }\end{array}$ & 19.656455 & 73.152646 & 51 \\
\hline \multirow[t]{2}{*}{24} & \multirow[t]{2}{*}{ Capnodium sp.1 } & Ficus racemosa $\mathrm{L}$. & 201010 & 16.10 .2012 & $\begin{array}{l}\text { On the way to } \\
\text { Zap, Greater } \\
\text { Thane }\end{array}$ & 19.898442 & 73.228724 & 375 \\
\hline & & Psidium guajava L. & 196490 & 28.09 .2013 & $\begin{array}{l}\text { Phansad WLS, } \\
\text { Raigad Dist. }\end{array}$ & 18.424063 & 72.949255 & 239 \\
\hline \multirow[t]{6}{*}{25} & \multirow[t]{6}{*}{ Cercospora apii Fresen } & $\begin{array}{l}\text { Colocasia esculenta }(\mathrm{L} .) \\
\text { Schott }\end{array}$ & 201089 & 17.10 .2012 & $\begin{array}{l}\text { On the way to } \\
\text { Dahanu, Greater } \\
\text { Thane }\end{array}$ & 19.596222 & 72.912617 & 92 \\
\hline & & Poaceae species 1 & 196371 & 24.09 .2013 & $\begin{array}{l}\text { On the way to } \\
\text { Sanjay Gandhi } \\
\text { National Park, } \\
\text { Greater Thane }\end{array}$ & 19.398960 & 72.885344 & 71 \\
\hline & & Fern & 196374 & 24.09 .2013 & $\begin{array}{l}\text { On the way to } \\
\text { Sanjay Gandhi } \\
\text { National Park, } \\
\text { Greater Thane }\end{array}$ & 19.398960 & 72.885344 & 71 \\
\hline & & Alternanthera $\mathrm{sp}$. & 201130 & 18.10 .2012 & $\begin{array}{l}\text { Pulachiwadi, } \\
\text { Greater Thane } \\
\end{array}$ & 19.880359 & 73.389547 & 395 \\
\hline & & Arisaema sp. & 201030 & 16.10 .2012 & $\begin{array}{l}\text { Shirpur, Greater } \\
\text { Thane }\end{array}$ & 19.261153 & 73.605987 & 148 \\
\hline & & Blumea sp. & 201040 & 16.10 .2012 & $\begin{array}{l}\text { Shirpur, Greater } \\
\text { Thane }\end{array}$ & 19.261153 & 73.605987 & 148 \\
\hline
\end{tabular}


Table 1 Continued.

\begin{tabular}{|c|c|c|c|c|c|c|c|c|}
\hline $\begin{array}{l}\text { Sr. } \\
\text { No. }\end{array}$ & Fungi & Host plants & $\begin{array}{l}\text { Collection } \\
\text { No. BSI } \\
\text { (WC) }\end{array}$ & $\begin{array}{l}\text { Date of } \\
\text { collection }\end{array}$ & Sampling sites & $\begin{array}{l}\text { Latitude } \\
\text { (N) }\end{array}$ & $\begin{array}{l}\text { Longitude } \\
\text { (E) }\end{array}$ & $\begin{array}{l}\text { Altitude } \\
\text { (m) }\end{array}$ \\
\hline & & Amorphophallus sp. & 201170 & 19.10.2012 & $\begin{array}{l}\text { Tansa WLS, } \\
\text { Greater Thane }\end{array}$ & 19.529328 & 73.258630 & 295 \\
\hline 26 & Cercospora blumeicola S. Das & Blumea sp. & 201040 & 16.10 .2012 & $\begin{array}{l}\text { Shirpur, Greater } \\
\text { Thane }\end{array}$ & 19.261153 & 73.605987 & 148 \\
\hline 27 & $\begin{array}{l}\text { Cercospora careyae } \\
\text { Ramakrishnan }\end{array}$ & Careya arborea Roxb. & 200062 & 20.01.2012 & $\begin{array}{l}\text { Sawantwadi, } \\
\text { Sindhudurg } \\
\text { Dist. }\end{array}$ & 15.904231 & 73.812647 & 184 \\
\hline 28 & $\begin{array}{l}\text { Cercospora ricinella Sacc. \& } \\
\text { Berl. }\end{array}$ & Euphorbia neriifolia $\mathrm{L}$. & 201074 & 17.10.2012 & $\begin{array}{l}\text { On the way to } \\
\text { Dahanu, Greater } \\
\text { Thane }\end{array}$ & 19.596222 & 72.912617 & 92 \\
\hline 29 & Cercosporella thunbergiae Hansf & Thunbergia sp. & 200002 & 20.01.2012 & $\begin{array}{l}\text { Akeri, } \\
\text { Sawantwadi, } \\
\text { Sindhudurg } \\
\text { Dist. }\end{array}$ & 15.937989 & 73.765437 & 66 \\
\hline 30 & Chalara siamense Pinnoi & $\begin{array}{l}\text { Pongamia pinnata }(\mathrm{L} .) \\
\text { Pierre }\end{array}$ & 196396 & 25.09 .2013 & $\begin{array}{l}\text { Sanjay Gandhi } \\
\text { National Park, } \\
\text { Greater Thane }\end{array}$ & 19.195361 & 72.906868 & 86 \\
\hline \multirow[t]{2}{*}{31} & \multirow[t]{2}{*}{ Chloridium indicum Subram. } & Mangifera indica $\mathrm{L}$. & 200220 & 22.01.2012 & $\begin{array}{l}\text { Amboli Ghat, } \\
\text { Sindhudurg } \\
\text { Dist. }\end{array}$ & 15.964716 & 74.003571 & 700 \\
\hline & & Mangifera indica $\mathrm{L}$. & 201056 & 16.10 .2012 & $\begin{array}{l}\text { Jawhar, Greater } \\
\text { Thane }\end{array}$ & 19.917037 & 73.222610 & 433 \\
\hline 32 & $\begin{array}{l}\text { Circinotrichum olivaceum (Speg.) } \\
\text { Piroz }\end{array}$ & Mangifera indica $\mathrm{L}$. & 201029 & 16.10 .2012 & $\begin{array}{l}\text { On the way to } \\
\text { Zap, Greater } \\
\text { Thane }\end{array}$ & 19.898442 & 73.228724 & 375 \\
\hline 33 & Cirsosia vateriae Hosagoudar & Unidentified plant species 4 & 199600 & 27.09 .2011 & $\begin{array}{l}\text { Matheran, } \\
\text { Raigad Dist. }\end{array}$ & 18.963105 & 73.264070 & 742 \\
\hline 34 & $\begin{array}{l}\text { Cladosporium cladosporioides } \\
\text { (Fresen.) G.A. de Vries }\end{array}$ & Hibiscus esculentus L. & 200228 & 22.01 .2012 & $\begin{array}{l}\text { Amboli Ghat, } \\
\text { Sindhudurg } \\
\text { Dist. }\end{array}$ & 15.964716 & 74.003571 & 700 \\
\hline
\end{tabular}


Table 1 Continued.

\begin{tabular}{|c|c|c|c|c|c|c|c|c|}
\hline $\begin{array}{l}\text { Sr. } \\
\text { No. }\end{array}$ & Fungi & Host plants & $\begin{array}{l}\text { Collection } \\
\text { No. BSI } \\
\text { (WC) }\end{array}$ & $\begin{array}{l}\text { Date of } \\
\text { collection }\end{array}$ & Sampling sites & $\begin{array}{l}\text { Latitude } \\
\text { (N) }\end{array}$ & $\begin{array}{l}\text { Longitude } \\
\text { (E) }\end{array}$ & $\begin{array}{l}\text { Altitude } \\
\text { (m) }\end{array}$ \\
\hline & & Coix lacryma-jobi $\mathrm{L}$. & 201005 & 16.10 .2012 & $\begin{array}{l}\text { On the way to } \\
\text { Zap, Greater } \\
\text { Thane }\end{array}$ & 19.898442 & 73.228724 & 375 \\
\hline \multirow[t]{6}{*}{35} & \multirow[t]{6}{*}{ Cladosporium colocasiae Sawada } & Eupatorium sp. & 200204 & 22.01 .2012 & $\begin{array}{l}\text { Amboli Ghat, } \\
\text { Sindhudurg } \\
\text { Dist. }\end{array}$ & 15.964716 & 74.003571 & 700 \\
\hline & & $\begin{array}{l}\text { Clerodendrum } \\
\text { grandiflorum (Hook.) } \\
\text { Schauer }\end{array}$ & 200214 & 22.01 .2012 & $\begin{array}{l}\text { Amboli Ghat, } \\
\text { Sindhudurg } \\
\text { Dist. }\end{array}$ & 15.964716 & 74.003571 & 700 \\
\hline & & Lygodium sp. & 200216 & 22.01 .2012 & $\begin{array}{l}\text { Amboli Ghat, } \\
\text { Sindhudurg } \\
\text { Dist. }\end{array}$ & 15.964716 & 74.003571 & 700 \\
\hline & & Blumea sp. & 200223 & 22.01 .2012 & $\begin{array}{l}\text { Amboli Ghat, } \\
\text { Sindhudurg } \\
\text { Dist. }\end{array}$ & 15.964716 & 74.003571 & 700 \\
\hline & & $\begin{array}{l}\text { Thysanolaena latifolia } \\
\text { (Roxb. ex Hornem.) Honda }\end{array}$ & 200156 & 21.01 .2012 & $\begin{array}{l}\text { Kesari, } \\
\text { Sindhudurg } \\
\text { Dist. } \\
\end{array}$ & 15.917294 & 73.941860 & 165 \\
\hline & & Hibiscus rosa-sinensis $\mathrm{L}$. & 200907 & 22.01 .2013 & $\begin{array}{l}\text { Near Amba } \\
\text { Ghat,Ratnagiri }\end{array}$ & 17.003574 & 73.762144 & 352 \\
\hline 36 & Cladosporium gallicola B. Sutton & Cordia sp. & 201083 & 17.10 .2012 & $\begin{array}{l}\text { On the way to } \\
\text { Dahanu, Greater } \\
\text { Thane }\end{array}$ & 19.596222 & 72.912617 & 92 \\
\hline \multirow[t]{2}{*}{37} & \multirow[t]{2}{*}{$\begin{array}{l}\text { Cladosporium oxysporum Berk. } \\
\& \text { Curt. }\end{array}$} & Cassia sp. & 200233 & 22.01 .2012 & $\begin{array}{l}\text { Amboli Ghat, } \\
\text { Sindhudurg } \\
\text { Dist. }\end{array}$ & 15.964716 & 74.003571 & 700 \\
\hline & & Hibiscus trionum $\mathrm{L}$. & 200050 & 20.01 .2012 & $\begin{array}{l}\text { Sawantwadi, } \\
\text { Sindhudurg } \\
\text { Dist. }\end{array}$ & 15.904231 & 73.812647 & 184 \\
\hline 38 & $\begin{array}{l}\text { Cladosporium spongiosum Berk. } \\
\& \text { M.A. Curtis }\end{array}$ & Cladosporium spongiosum & 200241 & 22.01 .2012 & $\begin{array}{l}\text { Vengurla, } \\
\text { Sindhudurg } \\
\text { Dist. }\end{array}$ & 15.863050 & 73.642144 & 11 \\
\hline
\end{tabular}


Table 1 Continued.

\begin{tabular}{|c|c|c|c|c|c|c|c|c|}
\hline $\begin{array}{l}\text { Sr. } \\
\text { No. }\end{array}$ & Fungi & Host plants & $\begin{array}{l}\text { Collection } \\
\text { No. BSI } \\
\text { (WC) } \\
\end{array}$ & $\begin{array}{l}\text { Date of } \\
\text { collection }\end{array}$ & Sampling sites & $\begin{array}{l}\text { Latitude } \\
\text { (N) }\end{array}$ & $\begin{array}{l}\text { Longitude } \\
\text { (E) }\end{array}$ & $\begin{array}{l}\text { Altitude } \\
\text { (m) }\end{array}$ \\
\hline \multirow[t]{4}{*}{39} & \multirow[t]{4}{*}{$\begin{array}{l}\text { Colletotrichum dematium (Pers.) } \\
\text { Grove }\end{array}$} & Euphorbiaceae & 196358 & 24.09 .2013 & $\begin{array}{l}\text { Karjat, Raigad } \\
\text { Dist. }\end{array}$ & 18.928594 & 73.432723 & 122 \\
\hline & & $\begin{array}{l}\text { Unidentified Poaceae } \\
\text { species } 2\end{array}$ & 199580 & 27.09 .2011 & $\begin{array}{l}\text { Karjat, Raigad } \\
\text { Dist. } \\
\end{array}$ & 18.928594 & 73.432723 & 122 \\
\hline & & $\begin{array}{l}\text { Unidentified Poaceae } \\
\text { species1 }\end{array}$ & 196371 & 24.09 .2013 & $\begin{array}{l}\text { On the way to } \\
\text { Sanjay Gandhi } \\
\text { National Park, } \\
\text { Greater Thane }\end{array}$ & 19.398960 & 72.885344 & 71 \\
\hline & & $\begin{array}{l}\text { Eleusine coracana }(\mathrm{L} .) \\
\text { Gaertn. }\end{array}$ & 201123 & 18.10 .2012 & $\begin{array}{l}\text { Pulachiwadi, } \\
\text { Greater Thane }\end{array}$ & 19.880359 & 73.389547 & 395 \\
\hline \multirow[t]{6}{*}{40} & \multirow[t]{6}{*}{$\begin{array}{l}\text { Colletotrichum gleosporioides } \\
\text { (Penz.) Penz. and Sacc }\end{array}$} & Careya arborea Roxb. & 200009 & 20.01 .2012 & \begin{tabular}{|l|} 
Akeri, \\
Sawantwadi \\
(Sindhudurg ) \\
\end{tabular} & 15.937989 & 73.765437 & 66 \\
\hline & & Gnetum ula Brongn. & 200174 & 22.01 .2012 & $\begin{array}{l}\text { Amboli Ghat, } \\
\text { Sindhudurg } \\
\text { Dist. } \\
\end{array}$ & 15.964716 & 74.003571 & 700 \\
\hline & & Persea sp. & 200190 & 22.01 .2012 & $\begin{array}{l}\text { Amboli Ghat, } \\
\text { Sindhudurg } \\
\text { Dist. } \\
\end{array}$ & 15.964716 & 74.003571 & 700 \\
\hline & & Ficus benghalensis L. & 194035 & 25.01 .2013 & $\begin{array}{l}\text { On the way to } \\
\text { Khed, Ratnagiri } \\
\text { Dist. }\end{array}$ & 17.702558 & 73.405491 & 125 \\
\hline & & Meyna laxiflora Robyns & 201018 & 18.10 .2012 & $\begin{array}{l}\text { On the way to } \\
\text { Zap, Greater } \\
\text { Thane } \\
\end{array}$ & 19.898442 & 73.228724 & 375 \\
\hline & & Erythrina sp. & 201136 & 18.10 .2012 & $\begin{array}{l}\text { Pulachiwadi, } \\
\text { Greater Thane }\end{array}$ & 19.880359 & 73.389547 & 395 \\
\hline 41 & $\begin{array}{l}\text { Colletotrichum lindemuthianum } \\
\text { (Sacc. \& Magnus) Briosi \& } \\
\text { Cavara }\end{array}$ & Dalbergia latifolia Roxb. & 200998 & 24.01 .2013 & $\begin{array}{l}\text { Kodawali- } \\
\text { Rajapura, } \\
\text { Ratnagiri }\end{array}$ & 16.682105 & 73.544952 & 167 \\
\hline
\end{tabular}


Table 1 Continued.

\begin{tabular}{|c|c|c|c|c|c|c|c|c|}
\hline $\begin{array}{l}\text { Sr. } \\
\text { No. }\end{array}$ & Fungi & Host plants & $\begin{array}{l}\text { Collection } \\
\text { No. BSI } \\
\text { (WC) }\end{array}$ & $\begin{array}{l}\text { Date of } \\
\text { collection }\end{array}$ & Sampling sites & $\begin{array}{l}\text { Latitude } \\
\text { (N) }\end{array}$ & $\begin{array}{l}\text { Longitude } \\
\text { (E) }\end{array}$ & $\begin{array}{l}\text { Altitude } \\
\text { (m) }\end{array}$ \\
\hline & & Carissa spinarum $\mathrm{L}$. & 201020 & 16.10 .2012 & $\begin{array}{l}\text { On the way to } \\
\text { Zap, Greater } \\
\text { Thane }\end{array}$ & 19.898442 & 73.228724 & 375 \\
\hline & & Meyna laxiflora Robyns & 201120 & 18.10 .2012 & $\begin{array}{l}\text { Pulachiwadi, } \\
\text { Greater Thane }\end{array}$ & 19.880359 & 73.389547 & 395 \\
\hline 42 & Coniothyrium palmarum Corda & $\begin{array}{l}\text { Roystonea regia (Kunth) } \\
\text { O.F.Cook }\end{array}$ & 196344 & 23.09 .2013 & $\begin{array}{l}\text { Matheran, } \\
\text { Raigad Dist. }\end{array}$ & 18.963105 & 73.264070 & 742 \\
\hline \multirow[t]{7}{*}{43} & \multirow[t]{7}{*}{$\begin{array}{l}\text { Corynespora cassicola (Berk \& } \\
\text { Curt.) Wei }\end{array}$} & $\begin{array}{l}\text { Hygrophila auriculata } \\
\text { (Schumach.) Heine }\end{array}$ & 200016 & 20.01 .2012 & $\begin{array}{l}\text { Akeri, } \\
\text { Sawantwadi, } \\
\text { Sindhudurg } \\
\text { Dist. }\end{array}$ & 15.937989 & 73.765437 & 66 \\
\hline & & $\begin{array}{l}\text { Clerodendrum } \\
\text { grandiflorum (Hook.) } \\
\text { Schauer }\end{array}$ & 200029 & 20.01 .2012 & $\begin{array}{l}\text { Akeri, } \\
\text { Sawantwadi, } \\
\text { Sindhudurg } \\
\text { Dist. }\end{array}$ & 15.937989 & 73.765437 & 66 \\
\hline & & Sida cordifolia $\mathrm{L}$. & 200034 & 20.01 .2012 & $\begin{array}{l}\text { Akeri, } \\
\text { Sawantwadi, } \\
\text { Sindhudurg } \\
\text { Dist. } \\
\end{array}$ & 15.937989 & 73.765437 & 66 \\
\hline & & $\begin{array}{l}\text { Clerodendrum } \\
\text { grandiflorum (Hook.) } \\
\text { Schauer }\end{array}$ & 200214 & 22.01 .2012 & $\begin{array}{l}\text { Amboli Ghat, } \\
\text { Sindhudurg } \\
\text { Dist. }\end{array}$ & 15.964716 & 74.003571 & 700 \\
\hline & & Dillenia pentagyna Roxb. & 200145 & 21.01 .2012 & $\begin{array}{l}\text { Fanaswadi, } \\
\text { Sindhudurg } \\
\text { Dist. }\end{array}$ & 15.890990 & 73.933043 & 151 \\
\hline & & Laportea sp. & 199582 & 27.09 .2011 & $\begin{array}{l}\text { Karjat, Raigad } \\
\text { Dist. }\end{array}$ & 18.928594 & 73.432723 & 122 \\
\hline & & $\begin{array}{l}\text { Eupatorium sp. } \\
\text { Pogostomon sp. }\end{array}$ & $\begin{array}{l}199589 \\
200110\end{array}$ & $\begin{array}{l}27.09 .2011 \\
21.01 .2012\end{array}$ & $\begin{array}{l}\text { Karjat, Raigad } \\
\text { Dist. } \\
\text { Kesari, } \\
\text { Sindhudurg } \\
\text { Dist. }\end{array}$ & $\begin{array}{r}18.928594 \\
15.917294\end{array}$ & $\begin{array}{l}73.432723 \\
73.941860\end{array}$ & $\begin{array}{l}122 \\
165\end{array}$ \\
\hline
\end{tabular}


Table 1 Continued.

\begin{tabular}{|c|c|c|c|c|c|c|c|c|}
\hline $\begin{array}{l}\text { Sr. } \\
\text { No. }\end{array}$ & Fungi & Host plants & $\begin{array}{l}\text { Collection } \\
\text { No. BSI } \\
\text { (WC) } \\
\end{array}$ & $\begin{array}{l}\text { Date of } \\
\text { collection }\end{array}$ & Sampling sites & $\begin{array}{l}\text { Latitude } \\
\text { (N) }\end{array}$ & $\begin{array}{l}\text { Longitude } \\
\text { (E) }\end{array}$ & $\begin{array}{l}\text { Altitude } \\
\text { (m) }\end{array}$ \\
\hline & & $\begin{array}{l}\text { Dregea volubilis (L.f.) } \\
\text { Benth. ex Hook.f. }\end{array}$ & 200115 & 21.01 .2012 & $\begin{array}{l}\text { Kesari, } \\
\text { Sindhudurg } \\
\text { Dist. } \\
\end{array}$ & 15.917294 & 73.941860 & 165 \\
\hline & & $\begin{array}{l}\text { Thysanolaena latifolia } \\
\text { (Roxb. ex Hornem.) Honda }\end{array}$ & 200149 & 21.01 .2012 & \begin{tabular}{|l} 
Kesari, \\
Sindhudurg \\
Dist. \\
\end{tabular} & 15.917294 & 73.941860 & 165 \\
\hline & & Cassia sp. & 199590 & 27.09 .2011 & $\begin{array}{l}\text { Khopoli, Raigad } \\
\text { Dist. }\end{array}$ & 18.798914 & 73.356470 & 115 \\
\hline & & Diospyros sp. & 199575 & 27.09 .2011 & $\begin{array}{l}\text { On the way to } \\
\text { Matheran, } \\
\text { Raigad Dist. }\end{array}$ & 19.009706 & 73.309401 & 134 \\
\hline 44 & $\begin{array}{l}\text { Corynespora torulosa (Syd. \& } \\
\text { P. Syd.) Crous }\end{array}$ & $\begin{array}{l}\text { Ensete superbum } \\
\text { Cheesm. }\end{array}$ & 194227 & 18.10 .2012 & \begin{tabular}{|l|} 
Pulachiwadi, \\
Greater Thane \\
\end{tabular} & 19.880359 & 73.389547 & 395 \\
\hline 45 & $\begin{array}{l}\text { Custingophora olivacea Stolk, } \\
\text { Hennebert \& Klopotek }\end{array}$ & $\begin{array}{l}\text { Clerodendrum } \\
\text { grandiflorum (Hook.) } \\
\text { Schauer }\end{array}$ & 200214 & 22.01 .2012 & $\begin{array}{l}\text { Amboli Ghat, } \\
\text { Sindhudurg } \\
\text { Dist. }\end{array}$ & 15.964716 & 74.003571 & 700 \\
\hline 46 & $\begin{array}{l}\text { Custingophora ratnagiriensis } \\
\text { Dubey \& Moonambeth }\end{array}$ & $\begin{array}{l}\text { Ziziphus oenopolia (L.) } \\
\text { Mill. }\end{array}$ & 200927 & 23.01 .2013 & \begin{tabular}{|l} 
Panval, \\
Ratnagiri Dist.
\end{tabular} & 17.003259 & 73.412566 & 112 \\
\hline 47 & $\begin{array}{l}\text { Deightoniella jabalpurensis G.P. } \\
\text { Agarwal \& Hasija }\end{array}$ & Apluda sp. & 201122 & 18.10 .2012 & $\begin{array}{l}\text { Pulachiwadi, } \\
\text { Greater Thane }\end{array}$ & 19.880359 & 73.389547 & 395 \\
\hline 48 & $\begin{array}{l}\text { Dendryphiella vinosa (Berk. \& } \\
\text { M.A. Curtis) Reisinger }\end{array}$ & Dillenia pentagyna Roxb. & 200145 & 21.01 .2012 & $\begin{array}{l}\text { Fanaswadi, } \\
\text { Sindhudurg } \\
\text { Dist. } \\
\end{array}$ & 15.890990 & 73.933043 & 151 \\
\hline 49 & $\begin{array}{l}\text { Dendryphion state of Pleospora } \\
\text { papaveracea (De Not.) Sacc }\end{array}$ & Dillenia pentagyna Roxb. & 200145 & 22.01 .2012 & $\begin{array}{l}\text { Fanaswadi, } \\
\text { Sindhudurg } \\
\text { Dist. } \\
\end{array}$ & 15.890990 & 73.933043 & 151 \\
\hline 50 & $\begin{array}{l}\text { Dictyosporium subramanianii B. } \\
\text { Sutton }\end{array}$ & $\begin{array}{l}\text { Roystonea regia (Kunth) } \\
\text { O.F.Cook }\end{array}$ & 196344 & 23.09 .2013 & $\begin{array}{l}\text { Matheran, } \\
\text { Raigad Dist. }\end{array}$ & 18.963105 & 73.264070 & 742 \\
\hline 51 & Didymella fabae Jellis \& Punith. & Tectona grandis $\mathrm{L}$. & 200037 & 20.01 .2012 & $\begin{array}{l}\text { Akeri, } \\
\text { Sawantwadi, } \\
\text { Sindhudurg } \\
\text { Dist. } \\
\end{array}$ & 15.937989 & 73.765437 & 66 \\
\hline
\end{tabular}


Table 1 Continued.

\begin{tabular}{|c|c|c|c|c|c|c|c|c|}
\hline $\begin{array}{l}\text { Sr. } \\
\text { No. }\end{array}$ & Fungi & Host plants & $\begin{array}{l}\text { Collection } \\
\text { No. BSI } \\
\text { (WC) }\end{array}$ & $\begin{array}{l}\text { Date of } \\
\text { collection }\end{array}$ & Sampling sites & $\begin{array}{l}\text { Latitude } \\
(\mathbf{N})\end{array}$ & $\begin{array}{l}\text { Longitude } \\
\text { (E) }\end{array}$ & $\begin{array}{l}\text { Altitude } \\
\text { (m) }\end{array}$ \\
\hline & & Ficus sp. & 200098 & 21.01.2012 & $\begin{array}{l}\text { Kesari, } \\
\text { Sindhudurg } \\
\text { Dist. }\end{array}$ & 15.917294 & 73.941860 & 165 \\
\hline 52 & Diplodia sp. & Agave americana $\mathrm{L}$. & 200243 & 22.1 .2012 & $\begin{array}{l}\text { Vengurla, } \\
\text { Sindhudurg } \\
\text { Dist. }\end{array}$ & 15.863050 & 73.642144 & 11 \\
\hline \multirow[t]{6}{*}{53} & \multirow[t]{6}{*}{$\begin{array}{l}\text { Domigoella asternarum Petr. \& } \\
\text { Shiff }\end{array}$} & Dillenia pentagyna Roxb. & 200145 & 21.01.2012 & $\begin{array}{l}\text { Fanaswadi, } \\
\text { Sindhudurg } \\
\text { Dist. }\end{array}$ & 15.890990 & 73.933043 & 151 \\
\hline & & Cocos nucifera $\mathrm{L}$. & 194061 & 26.01 .2013 & $\begin{array}{l}\text { Dapoli Forest } \\
\text { Range, Ratnagiri } \\
\text { Dist. }\end{array}$ & 17.786041 & 73.152662 & 60 \\
\hline & & Justicia adhatoda L. & 200078 & 21.01.2012 & $\begin{array}{l}\text { Kudal, } \\
\text { Sindhudurg } \\
\text { Dist. }\end{array}$ & 15.999956 & 73.683052 & 35 \\
\hline & & $\begin{array}{l}\text { Jasminum multiflorum } \\
\text { (Burm. f.) Andrews }\end{array}$ & 200080 & 21.01.2012 & $\begin{array}{l}\text { Kudal, } \\
\text { Sindhudurg } \\
\text { Dist. }\end{array}$ & 15.999956 & 73.683052 & 35 \\
\hline & & Woodfordia fruticosa Kurz. & 200973 & 24.01 .2013 & $\begin{array}{l}\text { Kurne-Lanja Tal } \\
\text { RF, Ratnagiri }\end{array}$ & 16.908281 & 73.508016 & 167 \\
\hline & & Malachra capitata $\mathrm{L}$. & 201161 & 19.10.2012 & $\begin{array}{l}\text { Tansa WLS, } \\
\text { Greater Thane }\end{array}$ & 19.529328 & 73.258630 & 295 \\
\hline \multirow[t]{3}{*}{54} & \multirow[t]{3}{*}{$\begin{array}{l}\text { Drechslera papendorfii (Aa) } \\
\text { M.B. Ellis }\end{array}$} & Poaceae species 4 & 196363 & 24.09 .2013 & $\begin{array}{l}\text { On the way to } \\
\text { Panvel, Raigad } \\
\text { Dist. }\end{array}$ & 18.953163 & 73.170934 & 34 \\
\hline & & $\begin{array}{l}\text { Eleusine coracana }(\mathrm{L} .) \\
\text { Gaertn. }\end{array}$ & 201123 & 18.10 .2012 & \begin{tabular}{|l|} 
Pulachiwadi \\
Greater Thane \\
\end{tabular} & 19.880359 & 73.389547 & 395 \\
\hline & & Ischaemum sp. & 201124 & 18.10.2012 & $\begin{array}{l}\text { Pulachiwadi, } \\
\text { Greater Thane }\end{array}$ & 19.880359 & 73.389547 & 395 \\
\hline 55 & Echidnodella polyalthiae Hosag. & Ixora sp. & 199601 & 27.09.2011 & $\begin{array}{l}\text { On the way to } \\
\text { Matheran, } \\
\text { Raigad Dist. }\end{array}$ & 19.009706 & 73.309401 & 134 \\
\hline
\end{tabular}


Table 1 Continued.

\begin{tabular}{|c|c|c|c|c|c|c|c|c|}
\hline $\begin{array}{l}\text { Sr. } \\
\text { No. }\end{array}$ & Fungi & Host plants & $\begin{array}{l}\text { Collection } \\
\text { No. BSI } \\
\text { (WC) }\end{array}$ & $\begin{array}{l}\text { Date of } \\
\text { collection }\end{array}$ & Sampling sites & $\begin{array}{l}\text { Latitude } \\
\text { (N) }\end{array}$ & $\begin{array}{l}\text { Longitude } \\
\text { (E) }\end{array}$ & $\begin{array}{l}\text { Altitude } \\
\text { (m) }\end{array}$ \\
\hline 56 & Epicoccum nigrum Link & Tectona grandis L.f. & 201009 & 16.10 .2012 & $\begin{array}{l}\text { On the way to } \\
\text { Zap, Greater } \\
\text { Thane }\end{array}$ & 19.898442 & 73.228724 & 375 \\
\hline 57 & $\begin{array}{l}\text { Excipulariopsis narsapurensis } \\
\text { Subram. }\end{array}$ & Cocos nucifera $\mathrm{L}$. & 194261 & 26.01 .2013 & $\begin{array}{l}\text { Dapoli, } \\
\text { Ratnagiri Dist. }\end{array}$ & 17.813476 & 73.300173 & 135 \\
\hline 58 & $\begin{array}{l}\text { Fusarium oxysporum E.F. Sm. \& } \\
\text { Swingle }\end{array}$ & Cocos nucifera $\mathrm{L}$. & 194061 & 26.01 .2013 & \begin{tabular}{|l|} 
Dapoli Forest \\
Range, Ratnagiri \\
Dist. \\
\end{tabular} & 17.786041 & 73.152662 & 60 \\
\hline 59 & Fusarium solani (Mart.) Sacc & Desmodium sp. & 199602 & 27.09 .2011 & $\begin{array}{l}\text { Matheran, } \\
\text { Raigad Dist. }\end{array}$ & 18.963105 & 73.264070 & 742 \\
\hline 60 & Fusicoccum sp. & $\begin{array}{l}\text { Calliandra haematocephala } \\
\text { Hassk. }\end{array}$ & 196373 & 24.09 .2013 & $\begin{array}{l}\text { On the way to } \\
\text { Sanjay Gandhi } \\
\text { National Park, } \\
\text { Greater Thane }\end{array}$ & 19.398960 & 72.885344 & 71 \\
\hline 61 & Gibberella baccata (Wallr.) Sacc. & Thunbergia sp. & 200002 & 20.01 .2012 & $\begin{array}{l}\text { Akeri, } \\
\text { Sawantwadi } \\
\text { (Sindhudurg ) }\end{array}$ & 15.937989 & 73.765437 & 66 \\
\hline 62 & Gleocladium penicilloides Corda. & Cocos nucifera $\mathrm{L}$. & 200161 & 21.01.2012 & $\begin{array}{l}\text { Kesari, } \\
\text { Sindhudurg } \\
\text { Dist. } \\
\end{array}$ & 15.917294 & 73.941860 & 165 \\
\hline 63 & $\begin{array}{l}\text { Glomerella cingulata (Stoneman) } \\
\text { Spauld. \& H. Schrenk }\end{array}$ & $\begin{array}{l}\text { Tylophora indica (Burm.f.) } \\
\text { Merr. }\end{array}$ & 200150 & 22.01 .2012 & $\begin{array}{l}\text { Kesari, } \\
\text { Sindhudurg } \\
\text { Dist. }\end{array}$ & 15.917294 & 73.941860 & 165 \\
\hline 64 & $\begin{array}{l}\text { Gonatophragmium mayteni S.K. } \\
\text { Singh, L.S. Yadav \& P.N. Singh }\end{array}$ & $\begin{array}{l}\text { Unidentified Leguminosae } \\
\text { sp. } 2\end{array}$ & 201028 & 16.10 .2012 & $\begin{array}{l}\text { On the way to } \\
\text { Zap, Greater } \\
\text { Thane }\end{array}$ & 19.898442 & 73.228724 & 375 \\
\hline 65 & $\begin{array}{l}\text { Goosiomyces bambusicola Dubey } \\
\text { \& Moonambeth }\end{array}$ & Bambusa bambos (L.) Voss & 200989 & 24.01 .2013 & $\begin{array}{l}\text { Kurne-Lanje Tal } \\
\text { Reserved Forest, } \\
\text { Ratnagiri }\end{array}$ & 16.908281 & 73.508016 & 167 \\
\hline
\end{tabular}


Table 1 Continued.

\begin{tabular}{|c|c|c|c|c|c|c|c|c|}
\hline $\begin{array}{l}\text { Sr. } \\
\text { No. }\end{array}$ & Fungi & Host plants & $\begin{array}{l}\text { Collection } \\
\text { No. BSI } \\
\text { (WC) }\end{array}$ & $\begin{array}{l}\text { Date of } \\
\text { collection }\end{array}$ & Sampling sites & $\begin{array}{l}\text { Latitude } \\
\text { (N) }\end{array}$ & $\begin{array}{l}\text { Longitude } \\
\text { (E) }\end{array}$ & $\begin{array}{l}\text { Altitude } \\
\text { (m) }\end{array}$ \\
\hline 66 & Graphium sp. & $\begin{array}{l}\text { Macaranga peltata (Roxb.) } \\
\text { Muell - Arg. }\end{array}$ & 201095 & 17.10.2012 & $\begin{array}{l}\text { On the way to } \\
\text { Dahanu, Greater } \\
\text { Thane }\end{array}$ & 19.596222 & 72.912617 & 92 \\
\hline 67 & $\begin{array}{l}\text { Hansfordiellopsis lichenicola } \\
\text { (Bat. \& H. Maia) Deighton }\end{array}$ & $\begin{array}{l}\text { Macaranga peltata (Roxb.) } \\
\text { Müll.Arg. }\end{array}$ & 200021 & 20.01 .2012 & $\begin{array}{l}\text { Akeri, } \\
\text { Sawantwadi, } \\
\text { Sindhudurg } \\
\text { Dist. } \\
\end{array}$ & 15.937989 & 73.765437 & 66 \\
\hline 68 & Harpographium sp. & $\begin{array}{l}\text { Dracena fragrans }(\mathrm{L} .) \mathrm{Ker} \\
\text { Gawl. }\end{array}$ & 194044 & 26.01 .2013 & $\begin{array}{l}\text { Khed, Ratnagiri } \\
\text { Dist. }\end{array}$ & 17.714175 & 73.385699 & 33 \\
\hline 69 & Helicoceras celtidis (Biv.) Linder & $\begin{array}{l}\text { Unidentified Leguminosae } \\
\text { sp. } 4\end{array}$ & 200209 & 22.01 .2012 & $\begin{array}{l}\text { Amboli Ghat, } \\
\text { Sindhudurg } \\
\text { Dist. }\end{array}$ & 15.964716 & 74.003571 & 700 \\
\hline 70 & $\begin{array}{l}\text { Helicomina costi M.A. Salam \& } \\
\text { P.N. Rao }\end{array}$ & Costus speciosus Konig & 196398 & 25.09 .2013 & \begin{tabular}{|l} 
Sanjay Gandhi \\
National Park, \\
Greater Thane \\
\end{tabular} & 19.195361 & 72.906868 & 86 \\
\hline 71 & $\begin{array}{l}\text { Helicosporium lumbricoides } \\
\text { Sacc. }\end{array}$ & Cocos nucifera $\mathrm{L}$. & 200099 & 21.01.2012 & $\begin{array}{l}\text { Kesari, } \\
\text { Sindhudurg } \\
\text { Dist. }\end{array}$ & 15.917294 & 73.941860 & 165 \\
\hline \multirow[t]{3}{*}{72} & \multirow[t]{3}{*}{ Isthmospora spinosa $\mathrm{F}$. Stevens } & $\begin{array}{l}\text { Holarrhena pubescens } \\
\text { (Buch. - Ham.) Wall. ex } \\
\text { DC. } \\
\end{array}$ & 194054 & 26.01 .2013 & $\begin{array}{l}\text { Dapoli Forest } \\
\text { Range, Ratnagiri } \\
\text { Dist. } \\
\end{array}$ & 17.786041 & 73.152662 & 60 \\
\hline & & Apocynaceae sp. 2 & 200148 & 21.01.2012 & $\begin{array}{l}\text { Kesari, } \\
\text { Sindhudurg } \\
\text { Dist. } \\
\end{array}$ & 15.917294 & 73.941860 & 165 \\
\hline & & $\begin{array}{l}\text { Tylophora indica (Burm.f.) } \\
\text { Merr. } \\
\text { Strychnos nux-vomica L. }\end{array}$ & $\begin{array}{l}200150 \\
200083\end{array}$ & $\begin{array}{l}21.01 .2012 \\
21.01 .2012\end{array}$ & $\begin{array}{l}\text { Kesari, } \\
\text { Sindhudurg } \\
\text { Dist. } \\
\text { Kudal, } \\
\text { Sindhudurg } \\
\text { Dist. }\end{array}$ & $\begin{array}{l}15.917294 \\
15.999956\end{array}$ & $\begin{array}{l}73.941860 \\
73.683052\end{array}$ & $\begin{array}{l}165 \\
35\end{array}$ \\
\hline
\end{tabular}


Table 1 Continued.

\begin{tabular}{|c|c|c|c|c|c|c|c|c|}
\hline $\begin{array}{l}\text { Sr. } \\
\text { No. }\end{array}$ & Fungi & Host plants & $\begin{array}{l}\text { Collection } \\
\text { No. BSI } \\
\text { (WC) }\end{array}$ & $\begin{array}{l}\text { Date of } \\
\text { collection }\end{array}$ & Sampling sites & $\begin{array}{l}\text { Latitude } \\
\text { (N) }\end{array}$ & $\begin{array}{l}\text { Longitude } \\
\text { (E) }\end{array}$ & $\begin{array}{l}\text { Altitude } \\
\text { (m) }\end{array}$ \\
\hline & & Volkameria inermis L. & 200085 & 21.01 .2012 & $\begin{array}{l}\text { Kudal, } \\
\text { Sindhudurg } \\
\text { Dist. } \\
\end{array}$ & 15.999956 & 73.683052 & 35 \\
\hline & & $\begin{array}{l}\text { Jasminum malabaricum } \\
\text { Wight. }\end{array}$ & 194067 & 26.01 .2013 & \begin{tabular}{|l|} 
Pachavali, \\
Dapoli, \\
Ratnagiri Dist. \\
\end{tabular} & 17.812811 & 73.285301 & 131 \\
\hline 73 & $\begin{array}{l}\text { Isthmospora state of } \\
\text { Trichothyrium asterophorum } \\
\text { (Berk. \& Broome) Höhn. }\end{array}$ & Mangifera indica $\mathrm{L}$. & 200141 & 21.01.2012 & $\begin{array}{l}\text { Fanaswadi, } \\
\text { Sindhudurg } \\
\text { Dist. } \\
\end{array}$ & 15.890990 & 73.933043 & 151 \\
\hline \multirow[t]{3}{*}{74} & \multirow[t]{3}{*}{ Khuskia oryzae H.J. Huds. } & $\begin{array}{l}\text { Thysanolaena latifolia } \\
\text { (Roxb. ex Hornem.) Honda }\end{array}$ & 200156 & 21.01.2012 & $\begin{array}{l}\text { Kesari, } \\
\text { Sindhudurg } \\
\text { Dist. } \\
\end{array}$ & 15.917294 & 73.941860 & 165 \\
\hline & & $\begin{array}{l}\text { Colocasia esculenta (L.) } \\
\text { Schott }\end{array}$ & 201089 & 17.10.2012 & $\begin{array}{l}\text { On the way to } \\
\text { Dahanu, Greater } \\
\text { Thane } \\
\end{array}$ & 19.596222 & 72.912617 & 92 \\
\hline & & $\begin{array}{l}\text { Unidentified Poaceae } \\
\text { species } 5\end{array}$ & 194274 & 24.01 .2013 & Ratnagiri & 17.006400 & 73.303397 & 54 \\
\hline \multirow[t]{2}{*}{75} & \multirow[t]{2}{*}{$\begin{array}{l}\text { Kirschsteiniothelia atra (Corda) } \\
\text { D. Hawksw }\end{array}$} & Apocynaceae sp. 2 & 200148 & 21.01.2012 & $\begin{array}{l}\text { Kesari, } \\
\text { Sindhudurg } \\
\text { Dist. }\end{array}$ & 15.917294 & 73.941860 & 165 \\
\hline & & $\begin{array}{l}\text { Jasminum multiflorum } \\
\text { (Burm. f.) Andrews }\end{array}$ & 200080 & 21.01.2012 & $\begin{array}{l}\text { Kudal, } \\
\text { Sindhudurg } \\
\text { Dist. }\end{array}$ & 15.999956 & 73.683052 & 35 \\
\hline 76 & $\begin{array}{l}\text { Leptoxyphium glochidion } \mathrm{H} . \\
\text { Yang \& K.D. Hyde }\end{array}$ & Terminalia elliptica Willd. & 201031 & 16.10 .2012 & $\begin{array}{l}\text { Shirpur, Greater } \\
\text { Thane }\end{array}$ & 19.261153 & 73.605987 & 148 \\
\hline 77 & Melanocarpus sp. & $\begin{array}{l}\text { Pandanus tectorius } \\
\text { Parkinson ex Du Roi }\end{array}$ & 200244 & & $\begin{array}{l}\text { Vengurla, } \\
\text { Sindhudurg } \\
\text { Dist. }\end{array}$ & 15.863050 & 73.642144 & 11 \\
\hline 78 & $\begin{array}{l}\text { Meliola allophyli-serrulati } \\
\text { Hosag. \& T.K. Abraham }\end{array}$ & Allophylus sp. & 200143 & 21.01.2012 & $\begin{array}{l}\text { Fanaswadi, } \\
\text { Sindhudurg } \\
\text { Dist. }\end{array}$ & 15.890990 & 73.933043 & 151 \\
\hline
\end{tabular}


Table 1 Continued.

\begin{tabular}{|c|c|c|c|c|c|c|c|c|}
\hline $\begin{array}{l}\text { Sr. } \\
\text { No. }\end{array}$ & Fungi & Host plants & $\begin{array}{l}\text { Collection } \\
\text { No. BSI } \\
\text { (WC) }\end{array}$ & $\begin{array}{l}\text { Date of } \\
\text { collection }\end{array}$ & Sampling sites & $\begin{array}{l}\text { Latitude } \\
\text { (N) }\end{array}$ & $\begin{array}{l}\text { Longitude } \\
\text { (E) }\end{array}$ & $\begin{array}{l}\text { Altitude } \\
\text { (m) }\end{array}$ \\
\hline 79 & Meliola alstoniae Koord & Unidentified plant sp. 8 & 200157 & 21.01.2012 & $\begin{array}{l}\text { Kesari, } \\
\text { Sindhudurg } \\
\text { Dist. }\end{array}$ & 15.917294 & 73.941860 & 165 \\
\hline 80 & Meliola bauhiniicola Yamam. & Bauhinia sp. & 200988 & 24.01 .2013 & $\begin{array}{l}\text { Kurne-Lanje Tal } \\
\text { RF, Ratnagiri }\end{array}$ & 16.908281 & 73.508016 & 167 \\
\hline 81 & $\begin{array}{l}\text { Meliola buteae Hafiz Khan, } \\
\text { Azmatullah \& Kafi }\end{array}$ & $\begin{array}{l}\text { Butea monosperma (Lam.) } \\
\text { Taub. }\end{array}$ & 196384 & 25.09 .2013 & \begin{tabular}{|l} 
Sanjay Gandhi \\
National Park, \\
Greater Thane \\
\end{tabular} & 19.195361 & 72.906868 & 86 \\
\hline 82 & $\begin{array}{l}\text { Meliola careyae (F. Stevens) } \\
\text { Hosag. }\end{array}$ & Careya arborea Roxb. & 200022 & 20.01 .2012 & $\begin{array}{l}\text { Akeri, } \\
\text { Sawantwadi, } \\
\text { Sindhudurg } \\
\text { Dist. }\end{array}$ & 15.937989 & 73.765437 & 66 \\
\hline 83 & $\begin{array}{l}\text { Meliola carissae var. spinari } \\
\text { Hosag. }\end{array}$ & Carissa spinarum $\mathrm{L}$. & 201027 & 16.10 .2012 & $\begin{array}{l}\text { On the way to } \\
\text { Zap, Greater } \\
\text { Thane }\end{array}$ & 19.898442 & 73.228724 & 375 \\
\hline 84 & $\begin{array}{l}\text { Meliola desmodii-triquetri Hosag. } \\
\& \text { Manojk }\end{array}$ & $\begin{array}{l}\text { Desmodium triflorum (L.) } \\
\text { DC }\end{array}$ & 200010 & 20.01 .2012 & $\begin{array}{l}\text { Akeri, } \\
\text { Sawantwadi } \\
\text { (Sindhudurg ) }\end{array}$ & 15.937989 & 73.765437 & 66 \\
\hline 85 & $\begin{array}{l}\text { Meliola eugeniae-jamboloidis } \\
\text { var. amphigena, A.K. Kar \& } \\
\text { Maity }\end{array}$ & Carissa spinarum $\mathrm{L}$. & 200084 & 21.01.2012 & $\begin{array}{l}\text { Kudal, } \\
\text { Sindhudurg } \\
\text { Dist. }\end{array}$ & 15.999956 & 73.683052 & 35 \\
\hline \multirow[t]{2}{*}{86} & \multirow[t]{2}{*}{ Meliola eugeniae-stocksii Hosag. } & Ixora brachiata Roxb. & 200178 & 22.01 .2012 & $\begin{array}{l}\text { Amboli Ghat, } \\
\text { Sindhudurg } \\
\text { Dist. } \\
\end{array}$ & 15.964716 & 74.003571 & 700 \\
\hline & & Ixora brachiata Roxb. & 200130 & 21.01.2012 & $\begin{array}{l}\text { Fanaswadi, } \\
\text { Sindhudurg } \\
\text { Dist. } \\
\end{array}$ & 15.890990 & 73.933043 & 151 \\
\hline 87 & Meliola hemidesmicola Hosag. & Apocynaceae sp. 2 & 200148 & 21.01.2012 & $\begin{array}{l}\text { Kesari, } \\
\text { Sindhudurg } \\
\text { Dist. }\end{array}$ & 15.917294 & 73.941860 & 165 \\
\hline
\end{tabular}


Table 1 Continued.

\begin{tabular}{|c|c|c|c|c|c|c|c|c|}
\hline $\begin{array}{l}\text { Sr. } \\
\text { No. }\end{array}$ & Fungi & Host plants & \begin{tabular}{|l|} 
Collection \\
No. BSI \\
(WC) \\
\end{tabular} & $\begin{array}{l}\text { Date of } \\
\text { collection }\end{array}$ & Sampling sites & $\begin{array}{l}\text { Latitude } \\
\text { (N) }\end{array}$ & $\begin{array}{l}\text { Longitude } \\
\text { (E) }\end{array}$ & $\begin{array}{l}\text { Altitude } \\
\text { (m) }\end{array}$ \\
\hline 88 & $\begin{array}{l}\text { Meliola holarhenae Hansf. \& } \\
\text { Thirum. }\end{array}$ & $\begin{array}{l}\text { Holarrhena pubescens } \\
\text { (Buch. - Ham) Wall. ex G. } \\
\text { Don }\end{array}$ & 196402 & 25.09 .2013 & $\begin{array}{l}\text { Sanjay Gandhi } \\
\text { National Park, } \\
\text { Greater Thane }\end{array}$ & 19.195361 & 72.906868 & 86 \\
\hline \multirow[t]{2}{*}{89} & \multirow[t]{2}{*}{ Meliola hyptidis Syd. \& P. Syd. } & Volkameria inermis $\mathrm{L}$. & 200085 & 21.01.2012 & $\begin{array}{l}\text { Kudal, } \\
\text { Sindhudurg } \\
\text { Dist. }\end{array}$ & 15.999956 & 73.683052 & 35 \\
\hline & & Plectranthus sp. & 196393 & 25.09 .2013 & $\begin{array}{l}\text { Sanjay Gandhi } \\
\text { National Park, } \\
\text { Greater Thane }\end{array}$ & 19.195361 & 72.906868 & 86 \\
\hline \multirow[t]{3}{*}{90} & \multirow[t]{3}{*}{$\begin{array}{l}\text { Meliola ixorae Yates var. } \\
\text { macrospora }\end{array}$} & Ixora brachiata Roxb. & 200180 & 22.01 .2012 & $\begin{array}{l}\text { Amboli Ghat, } \\
\text { Sindhudurg } \\
\text { Dist. }\end{array}$ & 15.964716 & 74.003571 & 700 \\
\hline & & Ixora brachiata Roxb. & 200215 & 22.01 .2012 & $\begin{array}{l}\text { Amboli Ghat, } \\
\text { Sindhudurg } \\
\text { Dist. }\end{array}$ & 15.964716 & 74.003571 & 700 \\
\hline & & Ixora coccinea $\mathrm{L}$. & 200077 & 21.01.2012 & $\begin{array}{l}\text { Kudal, } \\
\text { Sindhudurg } \\
\text { Dist. }\end{array}$ & 15.999956 & 73.683052 & 35 \\
\hline 91 & $\begin{array}{l}\text { Meliola ixorae-coccineae Hosag. } \\
\text { \& C.M. Pillai }\end{array}$ & Ixora brachiata Roxb. & 200180 & 22.01 .2012 & $\begin{array}{l}\text { Amboli Ghat, } \\
\text { Sindhudurg } \\
\text { Dist. }\end{array}$ & 15.964716 & 74.003571 & 700 \\
\hline \multirow[t]{2}{*}{92} & \multirow[t]{2}{*}{ Meliola jasminicola Henn. } & $\begin{array}{l}\text { Jasminum multiflorum } \\
\text { (Burm. f.) Andrews }\end{array}$ & 200080 & 21.01 .2012 & $\begin{array}{l}\text { Kudal, } \\
\text { Sindhudurg } \\
\text { Dist. }\end{array}$ & 15.999956 & 73.683052 & 35 \\
\hline & & Strychnos nux-vomica $\mathrm{L}$. & 200083 & 21.01 .2012 & $\begin{array}{l}\text { Kudal, } \\
\text { Sindhudurg } \\
\text { Dist. }\end{array}$ & 15.999956 & 73.683052 & 35 \\
\hline 93 & Meliola mangiferae Earle & Mangifera indica $\mathrm{L}$. & 200219 & 22.01 .2012 & $\begin{array}{l}\text { Amboli Ghat, } \\
\text { Sindhudurg } \\
\text { Dist. } \\
\end{array}$ & 15.964716 & 74.003571 & 700 \\
\hline 94 & $\begin{array}{l}\text { Meliola melanoxylonis Hosag. \& } \\
\text { C.M. Pillai }\end{array}$ & $\begin{array}{l}\text { Acacia auriculiformis } \\
\text { A.Cunn.ex Benth. }\end{array}$ & 200146 & 21.01 .2012 & $\begin{array}{l}\text { Kesari, } \\
\text { Sindhudurg }\end{array}$ & 15.917294 & 73.941860 & 165 \\
\hline
\end{tabular}


Table 1 Continued.

\begin{tabular}{|c|c|c|c|c|c|c|c|c|}
\hline $\begin{array}{l}\text { Sr. } \\
\text { No. }\end{array}$ & Fungi & Host plants & $\begin{array}{l}\text { Collection } \\
\text { No. BSI } \\
\text { (WC) }\end{array}$ & $\begin{array}{l}\text { Date of } \\
\text { collection }\end{array}$ & Sampling sites & $\begin{array}{l}\text { Latitude } \\
(\mathbf{N})\end{array}$ & $\begin{array}{l}\text { Longitude } \\
\text { (E) }\end{array}$ & $\begin{array}{l}\text { Altitude } \\
\text { (m) }\end{array}$ \\
\hline & & & & & Dist. & & & \\
\hline & & Carissa spinarum $\mathrm{L}$. & 200935 & 23.01 .2013 & Kirbet, Ratnagiri & 16.958657 & 73.744567 & 277 \\
\hline 95 & $\begin{array}{l}\text { Meliola mitragynae Syd. \& P. } \\
\text { Syd. }\end{array}$ & $\begin{array}{l}\text { Mitragyna parviflora } \\
\text { (Roxb.) Korth }\end{array}$ & 196410 & 25.09 .2013 & \begin{tabular}{|l} 
Sanjay Gandhi \\
National Park, \\
Greater Thane \\
\end{tabular} & 19.195361 & 72.906868 & 86 \\
\hline 96 & $\begin{array}{l}\text { Meliola pandanacearum Hosag. } \\
\& \text { T.K. Abraham }\end{array}$ & $\begin{array}{l}\text { Pandanus tectorius } \\
\text { Parkinson ex Du Roi }\end{array}$ & 200140 & 21.01.2012 & $\begin{array}{l}\text { Fanaswadi, } \\
\text { Sindhudurg } \\
\text { Dist. }\end{array}$ & 15.890990 & 73.933043 & 151 \\
\hline 97 & $\begin{array}{l}\text { Meliola pongamiae Hosag. \& } \\
\text { T.K. Abraham }\end{array}$ & $\begin{array}{l}\text { Pongamia pinnata }(\mathrm{L} .) \\
\text { Pierre }\end{array}$ & 200163 & 21.01.2012 & $\begin{array}{l}\text { Kesari, } \\
\text { Sindhudurg } \\
\text { Dist. } \\
\end{array}$ & 15.917294 & 73.941860 & 165 \\
\hline 98 & $\begin{array}{l}\text { Meliola semecarpi-anacardii } \\
\text { Hosag., Kaver., Raghu \& Goos }\end{array}$ & $\begin{array}{l}\text { Semecarpus anacardium } \\
\text { L.f. }\end{array}$ & 200158 & 21.01.2012 & $\begin{array}{l}\text { Kesari, } \\
\text { Sindhudurg } \\
\text { Dist. } \\
\end{array}$ & 15.917294 & 73.941860 & 165 \\
\hline 99 & Meliola tylophorae Hosag. & Tylophora sp. & 200038 & 20.01.2012 & $\begin{array}{l}\text { Akeri, } \\
\text { Sawantwadi, } \\
\text { Sindhudurg } \\
\text { Dist. } \\
\end{array}$ & 15.937989 & 73.765437 & 66 \\
\hline 100 & $\begin{array}{l}\text { Meliola tylophorae-indicae } \\
\text { Hosag. \& Manojk. }\end{array}$ & $\begin{array}{l}\text { Tylophora indica (Burm.f.) } \\
\text { Merr. }\end{array}$ & 200150 & 21.01.2012 & $\begin{array}{l}\text { Kesari, } \\
\text { Sindhudurg } \\
\text { Dist. }\end{array}$ & 15.917294 & 73.941860 & 165 \\
\hline 101 & $\begin{array}{l}\text { Meliola unonicola Hosag. \& T.K. } \\
\text { Abraham }\end{array}$ & Diospyros sp. & 196477 & 28.09 .2013 & $\begin{array}{l}\text { Phansad WLS, } \\
\text { Raigad Dist. }\end{array}$ & 18.424063 & 72.949255 & 239 \\
\hline 102 & Meliola ziziphi Hansf. \& Thirum. & Ziziphus jujuba Mill. & 200139 & 21.01.2012 & $\begin{array}{l}\text { Fanaswadi, } \\
\text { Sindhudurg } \\
\text { Dist. }\end{array}$ & 15.890990 & 73.933043 & 151 \\
\hline 103 & $\begin{array}{l}\text { Meliolina mollis (Berk. \& } \\
\text { Broome) Höhn }\end{array}$ & $\begin{array}{l}\text { Leea indica (Burm. f.) } \\
\text { Merr. }\end{array}$ & 200117 & 21.01 .2012 & $\begin{array}{l}\text { Kesari, } \\
\text { Sindhudurg } \\
\text { Dist. } \\
\end{array}$ & 15.917294 & 73.941860 & 165 \\
\hline
\end{tabular}


Table 1 Continued.

\begin{tabular}{|c|c|c|c|c|c|c|c|c|}
\hline $\begin{array}{l}\text { Sr. } \\
\text { No. }\end{array}$ & Fungi & Host plants & $\begin{array}{l}\text { Collection } \\
\text { No. BSI } \\
\text { (WC) }\end{array}$ & $\begin{array}{l}\text { Date of } \\
\text { collection }\end{array}$ & Sampling sites & $\begin{array}{l}\text { Latitude } \\
(\mathbf{N})\end{array}$ & $\begin{array}{l}\text { Longitude } \\
\text { (E) }\end{array}$ & $\begin{array}{l}\text { Altitude } \\
\text { (m) }\end{array}$ \\
\hline & & $\begin{array}{l}\text { Memecylon umbellatum } \\
\text { Burm f. }\end{array}$ & 200089 & 21.01.2012 & $\begin{array}{l}\text { Kudal, } \\
\text { Sindhudurg } \\
\text { Dist. }\end{array}$ & 15.999956 & 73.683052 & 35 \\
\hline 104 & $\begin{array}{l}\text { Metulocladosporiella musae } \\
\text { (E.W. Mason) Crous, Schroers, } \\
\text { J.Z. Groenew., U. Braun \& K. } \\
\text { Schub. }\end{array}$ & Cocos nucifera $\mathrm{L}$. & 200161 & 21.01.2012 & $\begin{array}{l}\text { Kesari, } \\
\text { Sindhudurg } \\
\text { Dist. }\end{array}$ & 15.917294 & 73.941860 & 165 \\
\hline 105 & $\begin{array}{l}\text { Microsphaeropsis sarcinellae } \\
\text { (V.P. Sahni) Morgan-Jones, }\end{array}$ & $\begin{array}{l}\text { Roystonea regia (Kunth) } \\
\text { O.F.Cook }\end{array}$ & 196344 & 23.09 .2013 & $\begin{array}{l}\text { Matheran, } \\
\text { Raigad }\end{array}$ & 18.963105 & 73.264070 & 742 \\
\hline 106 & Mitteriella zizyphina Syd. & Ziziphus jujuba Mill. & 201036 & 16.10 .2012 & $\begin{array}{l}\text { Shirpur, Greater } \\
\text { Thane }\end{array}$ & 19.261153 & 73.605987 & 148 \\
\hline 107 & $\begin{array}{l}\text { Moorella speciose P.Rag. Rao \& } \\
\text { D. Rao }\end{array}$ & Eugenia sp. & 194125 & 16.10 .2012 & $\begin{array}{l}\text { Wada,Greater } \\
\text { Thane }\end{array}$ & 19.656455 & 73.152646 & 51 \\
\hline \multirow[t]{2}{*}{108} & \multirow[t]{2}{*}{$\begin{array}{l}\text { Mycovellosiella solani-torvi } \\
\text { (Gonz. Frag. \& Cif.) Deighton }\end{array}$} & Grewia sp. & 200074 & 20.01.2012 & $\begin{array}{l}\text { Sawantwadi, } \\
\text { Sindhudurg } \\
\text { Dist. }\end{array}$ & 15.904231 & 73.812647 & 184 \\
\hline & & Vitex trifolia $\mathrm{L}$. & 200239 & 22.01 .2012 & $\begin{array}{l}\text { Vengurla, } \\
\text { Sindhudurg } \\
\text { Dist. }\end{array}$ & 15.863050 & 73.642144 & 11 \\
\hline \multirow[t]{2}{*}{109} & \multirow[t]{2}{*}{ Myrothecium roridum Tode } & Casearia sp. & 201033 & 16.10 .2012 & $\begin{array}{l}\text { Shirpur, Greater } \\
\text { Thane }\end{array}$ & 19.261153 & 73.605987 & 148 \\
\hline & & Bombax ceiba $\mathrm{L}$. & 201037 & 16.10 .2012 & $\begin{array}{l}\text { Shirpur, Greater } \\
\text { Thane }\end{array}$ & 19.261153 & 73.605987 & 148 \\
\hline \multirow[t]{2}{*}{110} & \multirow[t]{2}{*}{$\begin{array}{l}\text { Neopestalotiopsis asiatica } \\
\text { (Maharachch. \& K.D. Hyde) } \\
\text { Maharachch., K.D. Hyde \& Crous }\end{array}$} & Cocos nucifera $\mathrm{L}$. & 200045 & 20.01 .2012 & $\begin{array}{l}\text { Akeri, } \\
\text { Sawantwadi, } \\
\text { Sindhudurg } \\
\text { Dist. }\end{array}$ & 15.937989 & 73.765437 & 66 \\
\hline & & $\begin{array}{l}\text { Bombax ceiba } \mathrm{L} . \\
\text { Carissa spinarum } \mathrm{L} .\end{array}$ & $\begin{array}{l}201037 \\
201039\end{array}$ & $\begin{array}{l}16.10 .2012 \\
16.10 .2012\end{array}$ & $\begin{array}{l}\text { Shirpur, Greater } \\
\text { Thane } \\
\text { Shirpur, Greater } \\
\text { Thane }\end{array}$ & $\begin{array}{l}19.261153 \\
19.261153\end{array}$ & $\begin{array}{l}73.605987 \\
73.605987\end{array}$ & $\begin{array}{l}148 \\
148\end{array}$ \\
\hline
\end{tabular}


Table 1 Continued.

\begin{tabular}{|c|c|c|c|c|c|c|c|c|}
\hline $\begin{array}{l}\text { Sr. } \\
\text { No. }\end{array}$ & Fungi & Host plants & \begin{tabular}{|l|} 
Collection \\
No. BSI \\
(WC) \\
\end{tabular} & $\begin{array}{l}\text { Date of } \\
\text { collection }\end{array}$ & Sampling sites & $\begin{array}{l}\text { Latitude } \\
\text { (N) }\end{array}$ & $\begin{array}{l}\text { Longitude } \\
\text { (E) }\end{array}$ & $\begin{array}{l}\text { Altitude } \\
\text { (m) }\end{array}$ \\
\hline \multirow[t]{2}{*}{111} & \multirow[t]{2}{*}{$\begin{array}{l}\text { Nigrospora sphaerica (Sacc.) } \\
\text { E.W. Mason }\end{array}$} & Euphorbia neriifolia L. & 201074 & 17.10 .2012 & $\begin{array}{l}\text { On the way to } \\
\text { Dahanu, Greater } \\
\text { Thane }\end{array}$ & 19.596222 & 72.912617 & 92 \\
\hline & & $\begin{array}{l}\text { Eleusine coracana }(\mathrm{L} .) \\
\text { Gaertn. }\end{array}$ & 201123 & 18.10 .2012 & $\begin{array}{l}\text { Pulachiwadi, } \\
\text { Greater Thane }\end{array}$ & 19.880359 & 73.389547 & 395 \\
\hline 112 & $\begin{array}{l}\text { Passalora desmanthi (Ellis \& } \\
\text { Kellerm.) U. Braun }\end{array}$ & $\begin{array}{l}\text { Tinospora cordifolia } \\
\text { (Thunb.) Miers }\end{array}$ & 201082 & 17.10.2012 & $\begin{array}{l}\text { On the way to } \\
\text { Dahanu, Greater } \\
\text { Thane }\end{array}$ & 19.596222 & 72.912617 & 92 \\
\hline 113 & Penicillium notatum Westling & Careya arborea Roxb. & 194002 & 24.01 .2013 & $\begin{array}{l}\text { Rajapura, } \\
\text { Ratnagiri Dist. }\end{array}$ & 16.656979 & 73.519422 & 89 \\
\hline \multirow[t]{3}{*}{114} & \multirow[t]{3}{*}{ Periconia byssoides Pers. } & Thunbergia sp. & 200002 & 20.01 .2012 & $\begin{array}{l}\text { Akeri, } \\
\text { Sawantwadi } \\
\text { Tal.,Sindhudurg } \\
\text { Dist. } \\
\end{array}$ & 15.937989 & 73.765437 & 66 \\
\hline & & Eupatorium sp. & 200004 & 20.01 .2012 & $\begin{array}{l}\text { Akeri, } \\
\text { Sawantwadi, } \\
\text { Sindhudurg } \\
\text { Dist. }\end{array}$ & 15.937989 & 73.765437 & 66 \\
\hline & & Unidentified plant sp.9 & 200007 & 20.01 .2012 & $\begin{array}{l}\text { Akeri, } \\
\text { Sawantwadi, } \\
\text { Sindhudurg } \\
\text { Dist. }\end{array}$ & 15.937989 & 73.765437 & 66 \\
\hline 115 & $\begin{array}{l}\text { Periconia cambrensis E.W. } \\
\text { Mason \& M.B. Ellis }\end{array}$ & Bambusa bambos (L.) Voss & 196168 & & Raigad Forest & 18.146382 & 73.459228 & 277 \\
\hline 116 & $\begin{array}{l}\text { Periconia cookei E.W. Mason \& } \\
\text { M.B. Ellis }\end{array}$ & $\begin{array}{l}\text { Ipomoea pes-caprae (L.) } \\
\text { R.Br. }\end{array}$ & 194026 & 25.01 .2013 & $\begin{array}{l}\text { Ganpatipule, } \\
\text { Ratnagiri Dist. } \\
\text { Kesari, }\end{array}$ & 17.144616 & 73.268033 & 60 \\
\hline 117 & Periconia lateralis Ellis \& Everh, & Pogostomon sp. & 200110 & 21.01.2012 & $\begin{array}{l}\text { Sindhudurg } \\
\text { Dist. }\end{array}$ & 15.917294 & 73.941860 & 165 \\
\hline
\end{tabular}


Table 1 Continued.

\begin{tabular}{|c|c|c|c|c|c|c|c|c|}
\hline $\begin{array}{l}\text { Sr. } \\
\text { No. }\end{array}$ & Fungi & Host plants & $\begin{array}{l}\text { Collection } \\
\text { No. BSI } \\
\text { (WC) }\end{array}$ & $\begin{array}{l}\text { Date of } \\
\text { collection }\end{array}$ & Sampling sites & $\begin{array}{l}\text { Latitude } \\
\text { (N) }\end{array}$ & $\begin{array}{l}\text { Longitude } \\
\text { (E) }\end{array}$ & $\begin{array}{l}\text { Altitude } \\
\text { (m) }\end{array}$ \\
\hline & & $\begin{array}{l}\text { Pongamia pinnata }(\mathrm{L} .) \\
\text { Pierre }\end{array}$ & 196396 & 25.09 .2013 & $\begin{array}{l}\text { Sanjay Gandhi } \\
\text { National Park, } \\
\text { Greater Thane } \\
\end{array}$ & 19.195361 & 72.906868 & 86 \\
\hline 118 & Periconia minutissima Corda & $\begin{array}{l}\text { Eleusine coracana }(\mathrm{L} .) \\
\text { Gaertn. }\end{array}$ & 201123 & 18.10.2012 & \begin{tabular}{|l|} 
Pulachiwadi \\
Greater Thane \\
\end{tabular} & 19.880359 & 73.389547 & 395 \\
\hline 119 & $\begin{array}{l}\text { Periconiella telopeae (Hansf.) } \\
\text { M.B. Ellis }\end{array}$ & Unidentified plant sp. 8 & 200157 & 21.01.2012 & $\begin{array}{l}\text { Kesari, } \\
\text { Sindhudurg } \\
\text { Dist. }\end{array}$ & 15.917294 & 73.941860 & 165 \\
\hline 120 & $\begin{array}{l}\text { Pestalotiopsis anacardii Kamil et } \\
\text { al }\end{array}$ & Mangifera indica $\mathrm{L}$. & 200138 & 21.01.2012 & $\begin{array}{l}\text { Fanaswadi, } \\
\text { Sindhudurg } \\
\text { Dist. }\end{array}$ & 15.890990 & 73.933043 & 151 \\
\hline 121 & $\begin{array}{l}\text { Pestalotiopsis funerea } \\
\text { (Desmazieres) Steyaert }\end{array}$ & Leea indica (Burm. f.) Merr & 200046 & 20.01 .2012 & $\begin{array}{l}\text { Akeri, } \\
\text { Sawantwadi, } \\
\text { Sindhudurg } \\
\text { Dist. }\end{array}$ & 15.937989 & 73.765437 & 66 \\
\hline \multirow[t]{3}{*}{122} & \multirow[t]{3}{*}{$\begin{array}{l}\text { Pestalotiopsis linearis } \\
\text { Maharachch. \& K.D. Hyde }\end{array}$} & Mangifera indica $\mathrm{L}$. & 200219 & 22.01 .2012 & $\begin{array}{l}\text { Amboli Ghat, } \\
\text { Sindhudurg } \\
\text { Dist. }\end{array}$ & 15.964716 & 74.003571 & 700 \\
\hline & & $\begin{array}{l}\text { Glochidion velutinum } \\
\text { Wight }\end{array}$ & 200144 & 21.01.2012 & $\begin{array}{l}\text { Fanaswadi, } \\
\text { Sindhudurg } \\
\text { Dist. }\end{array}$ & 15.890990 & 73.933043 & 151 \\
\hline & & Acacia sp. & 200108 & 21.01.2012 & $\begin{array}{l}\text { Kesari, } \\
\text { Sindhudurg } \\
\text { Dist. }\end{array}$ & 15.917294 & 73.941860 & 165 \\
\hline 123 & $\begin{array}{l}\text { Phaeoisaria clematidis (Fuckel) } \\
\text { S. Hughes }\end{array}$ & $\begin{array}{l}\text { Roystonea regia (Kunth) } \\
\text { O.F.Cook }\end{array}$ & 196344 & 23.09 .2013 & $\begin{array}{l}\text { Matheran, } \\
\text { Raigad Dist. }\end{array}$ & 18.963105 & 73.264070 & 742 \\
\hline 124 & $\begin{array}{l}\text { Phialophora cyclaminis J.F.H. } \\
\text { Beyma }\end{array}$ & $\begin{array}{l}\text { Chrysalidocarpus lutescens } \\
\text { Wendl. }\end{array}$ & 201046 & 16.10 .2012 & $\begin{array}{l}\text { Jawhar, Greater } \\
\text { Thane }\end{array}$ & 19.917037 & 73.222610 & 433 \\
\hline 125 & Philonectria sp. & Dillenia pentagyna Roxb. & 200145 & 21.01.2012 & $\begin{array}{l}\text { Fanaswadi, } \\
\text { Sindhudurg } \\
\text { Dist. }\end{array}$ & 15.890990 & 73.933043 & 151 \\
\hline
\end{tabular}


Table 1 Continued.

\begin{tabular}{|c|c|c|c|c|c|c|c|c|}
\hline $\begin{array}{l}\text { Sr. } \\
\text { No. }\end{array}$ & Fungi & Host plants & $\begin{array}{l}\text { Collection } \\
\text { No. BSI } \\
\text { (WC) }\end{array}$ & $\begin{array}{l}\text { Date of } \\
\text { collection }\end{array}$ & Sampling sites & $\begin{array}{l}\text { Latitude } \\
\text { (N) }\end{array}$ & $\begin{array}{l}\text { Longitude } \\
\text { (E) }\end{array}$ & $\begin{array}{l}\text { Altitude } \\
\text { (m) }\end{array}$ \\
\hline 126 & $\begin{array}{l}\text { Phoma multirostrata Doretosch } \\
\& \text { Boerema }\end{array}$ & $\begin{array}{l}\text { Calliandra haematocephala } \\
\text { Hassk. }\end{array}$ & 196373 & 24.09 .2013 & $\begin{array}{l}\text { On the way to } \\
\text { Sanjay Gandhi } \\
\text { National Park, } \\
\text { Greater Thane }\end{array}$ & 19.398960 & 72.885344 & 71 \\
\hline 127 & $\begin{array}{l}\text { Phoma tropica Schneider \& } \\
\text { Boerema }\end{array}$ & Ficus sp. & 200159 & 21.01.2012 & $\begin{array}{l}\text { Kesari, } \\
\text { Sindhudurg } \\
\text { Dist. }\end{array}$ & 15.917294 & 73.941860 & 165 \\
\hline 128 & Phoma nebulosa (Pers.) Mont. & Ipomea sp. & 200053 & 20.01.2012 & $\begin{array}{l}\text { Sawantwadi, } \\
\text { Sindhudurg } \\
\text { Dist. }\end{array}$ & 15.904231 & 73.812647 & 184 \\
\hline 129 & Phyllachora sp. & $\begin{array}{l}\text { Ixora brachiata Roxb. ex } \\
\text { DC }\end{array}$ & 200996 & 24.01 .2013 & $\begin{array}{l}\text { Kodawali- } \\
\text { Rajapura, } \\
\text { Ratnagiri } \\
\end{array}$ & 16.682105 & 73.544952 & 167 \\
\hline 130 & $\begin{array}{l}\text { Pirozynskiella solanina (Sacc. \& } \\
\text { P. Syd.) Hughes }\end{array}$ & $\begin{array}{l}\text { Jasminum multiflorum } \\
\text { (Burm. f.) Andrews }\end{array}$ & 200080 & 21.01.2012 & \begin{tabular}{|l} 
Kudal, \\
Sindhudurg \\
Dist. \\
\end{tabular} & 15.999956 & 73.683052 & 35 \\
\hline 131 & $\begin{array}{l}\text { Pithomyces ellisii V.G. Rao \& } \\
\text { Chary }\end{array}$ & $\begin{array}{l}\text { Memecylon umbellatum } \\
\text { Burm. f. }\end{array}$ & 201013 & 16.10 .2012 & $\begin{array}{l}\text { On the way to } \\
\text { Zap, Greater } \\
\text { Thane }\end{array}$ & 19.898442 & 73.228724 & 375 \\
\hline 132 & $\begin{array}{l}\text { Pithomyces pavgii (V.R. Nath) } \\
\text { M.E. Palm, E.L. Stewart \& } \\
\text { Rossman }\end{array}$ & Bambusa bambos (L.) Voss & 196329 & 23.09 .2013 & $\begin{array}{l}\text { Matheran, } \\
\text { Raigad Dist. }\end{array}$ & 18.963105 & 73.264070 & 742 \\
\hline 133 & Pleurocytospora vestita Petr. & $\begin{array}{l}\text { Madhuca longifolia var. } \\
\text { latifolia (Roxb.) Chev }\end{array}$ & 201091 & 17.10 .2012 & $\begin{array}{l}\text { On the way to } \\
\text { Dahanu, Greater } \\
\text { Thane }\end{array}$ & 19.596222 & 72.912617 & 92 \\
\hline 134 & $\begin{array}{l}\text { Polytretophora calcarata } \\
\text { Mercado }\end{array}$ & Dendrocalamus sp. & 200133 & 21.01.2012 & $\begin{array}{l}\text { Fanaswadi, } \\
\text { Sindhudurg } \\
\text { Dist. }\end{array}$ & 15.890990 & 73.933043 & 151 \\
\hline 135 & Porrectotheca sp. & Bambusa bambos (L.) Voss & 200015 & 20.01 .2012 & $\begin{array}{l}\text { Akeri, } \\
\text { Sawantwadi, } \\
\text { Sindhudurg } \\
\text { Dist. } \\
\end{array}$ & 15.937989 & 73.765437 & 66 \\
\hline
\end{tabular}


Table 1 Continued.

\begin{tabular}{|c|c|c|c|c|c|c|c|c|}
\hline $\begin{array}{l}\text { Sr. } \\
\text { No. }\end{array}$ & Fungi & Host plants & $\begin{array}{l}\text { Collection } \\
\text { No. BSI } \\
\text { (WC) }\end{array}$ & $\begin{array}{l}\text { Date of } \\
\text { collection }\end{array}$ & Sampling sites & $\begin{array}{l}\text { Latitude } \\
\text { (N) }\end{array}$ & $\begin{array}{l}\text { Longitude } \\
\text { (E) }\end{array}$ & $\begin{array}{l}\text { Altitude } \\
\text { (m) }\end{array}$ \\
\hline 136 & Prathigada sp. & $\begin{array}{l}\text { Pandanus tectorius } \\
\text { Parkinson ex Du Roi }\end{array}$ & 200244 & 21.01.2012 & $\begin{array}{l}\text { Vengurla, } \\
\text { Sindhudurg } \\
\text { Dist. }\end{array}$ & 15.863050 & 73.642144 & 11 \\
\hline 137 & $\begin{array}{l}\text { Prathigada terminaliae (Syd.) B. } \\
\text { Sutton }\end{array}$ & Terminalia sp. & 200999 & 24.01 .2013 & $\begin{array}{l}\text { Kodawali- } \\
\text { Rajapura, } \\
\text { Ratnagiri }\end{array}$ & 16.682105 & 73.544952 & 167 \\
\hline 138 & $\begin{array}{l}\text { Prillieuxina polyalthiae Hosag. \& } \\
\text { T.K. Abraham }\end{array}$ & Desmodium sp. & 200041 & 20.01 .2012 & $\begin{array}{l}\text { Akeri, } \\
\text { Sawantwadi, } \\
\text { Sindhudurg } \\
\text { Dist. }\end{array}$ & 15.937989 & 73.765437 & 66 \\
\hline 139 & $\begin{array}{l}\text { Pseudocercospora conyzae } \\
\text { Sawada ex Goh \& W.H. Hsieh }\end{array}$ & Eupatorium sp. & 200004 & 20.01 .2012 & $\begin{array}{l}\text { Akeri, } \\
\text { Sawantwadi, } \\
\text { Sindhudurg }\end{array}$ & 15.937989 & 73.765437 & 66 \\
\hline 140 & $\begin{array}{l}\text { Pseudocercospora eupatorii- } \\
\text { formosanae U. Braun \& Bagyan. }\end{array}$ & Eupatorium sp. & 201045 & 16.10 .2012 & $\begin{array}{l}\text { Shirpur,Greater } \\
\text { Thane } \\
\end{array}$ & 19.261153 & 73.605987 & 148 \\
\hline 141 & Pseudocercospora sp. 1 & Pseudocercospora sp. 1 & 200020 & 20.01 .2012 & $\begin{array}{l}\text { Akeri, } \\
\text { Sawantwadi, } \\
\text { Sindhudurg } \\
\text { Dist. }\end{array}$ & 15.937989 & 73.765437 & 66 \\
\hline 142 & Puccinia kraussiana Cooke & Smilax sp. & 194033 & 25.01 .2013 & $\begin{array}{l}\text { On the way to } \\
\text { Khed, Ratnagiri } \\
\text { Dist. }\end{array}$ & 17.702558 & 73.405491 & 125 \\
\hline \multirow[t]{2}{*}{143} & \multirow[t]{2}{*}{$\begin{array}{l}\text { Puccinia phragmitis (Schumach.) } \\
\text { Körn. }\end{array}$} & Smilax sp. & 200056 & 20.01 .2012 & $\begin{array}{l}\text { Sawantwadi, } \\
\text { Sindhudurg } \\
\text { Dist. }\end{array}$ & 15.904231 & 73.812647 & 184 \\
\hline & & Smilax sp. & 200259 & 23.01 .2012 & $\begin{array}{l}\text { Vengurla, } \\
\text { Sindhudurg } \\
\text { Dist. }\end{array}$ & 15.863050 & 73.642144 & 11 \\
\hline 144 & Pyriculariopsis sp. & Commelina benghalensis L. & 201164 & 19.10 .2012 & $\begin{array}{l}\text { Tansa WLS, } \\
\text { Greater Thane }\end{array}$ & 19.529328 & 73.258630 & 295 \\
\hline
\end{tabular}


Table 1 Continued.

\begin{tabular}{|c|c|c|c|c|c|c|c|c|}
\hline $\begin{array}{l}\text { Sr. } \\
\text { No. }\end{array}$ & Fungi & Host plants & $\begin{array}{l}\text { Collection } \\
\text { No. BSI } \\
\text { (WC) }\end{array}$ & $\begin{array}{l}\text { Date of } \\
\text { collection }\end{array}$ & Sampling sites & $\begin{array}{l}\text { Latitude } \\
\text { (N) }\end{array}$ & $\begin{array}{l}\text { Longitude } \\
\text { (E) }\end{array}$ & $\begin{array}{l}\text { Altitude } \\
\text { (m) }\end{array}$ \\
\hline 145 & Questeriella strychni Hosag. & $\begin{array}{l}\text { Pongamia pinnata }(\mathrm{L} .) \\
\text { Pierre }\end{array}$ & 200163 & 21.01.2012 & $\begin{array}{l}\text { Kesari, } \\
\text { Sindhudurg } \\
\text { Dist. }\end{array}$ & 15.917294 & 73.941860 & 165 \\
\hline 146 & $\begin{array}{l}\text { Repetophragma ellisii (Piroz.) } \\
\text { R.F. Castañeda, McKenzie \& } \\
\text { K.D. Hyde }\end{array}$ & Bambusa bambos (L.) Voss & 200948 & 23.01 .2013 & Kirbet, Ratnagiri & 16.958657 & 73.744567 & 277 \\
\hline \multirow[t]{2}{*}{147} & \multirow[t]{2}{*}{ Rhinocladium sp. } & $\begin{array}{l}\text { Persicaria auriculata } \\
\text { (Makino) Masam. }\end{array}$ & 200167 & 22.01 .2012 & $\begin{array}{l}\text { Amboli Ghat, } \\
\text { Sindhudurg } \\
\text { Dist. } \\
\end{array}$ & 15.964716 & 74.003571 & 700 \\
\hline & & $\begin{array}{l}\text { Macaranga peltata (Roxb.) } \\
\text { Muell - Arg. }\end{array}$ & 194032 & 25.01 .2013 & $\begin{array}{l}\text { On the way to } \\
\text { Khed, Ratnagiri } \\
\text { Dist. }\end{array}$ & 17.702558 & 73.405491 & 125 \\
\hline 148 & $\begin{array}{l}\text { Sarcinella cassiae-fistulae Hosag. } \\
\text { \& Shajivaz }\end{array}$ & Cassia fistula $\mathrm{L}$. & 201065 & 17.10.2012 & $\begin{array}{l}\text { On the way to } \\
\text { Dahanu, Greater } \\
\text { Thane }\end{array}$ & 19.596222 & 72.912617 & 92 \\
\hline 149 & $\begin{array}{l}\text { Sarcinella gmelinae Hosag., } \\
\text { Archana, Harish, Riju \& D.K. } \\
\text { Agarwal }\end{array}$ & Tectona grandis L.f. & 201119 & 18.10 .2012 & $\begin{array}{l}\text { Pulachiwadi, } \\
\text { Greater Thane }\end{array}$ & 19.880359 & 73.389547 & 395 \\
\hline 150 & $\begin{array}{l}\text { Sarcinella gymnosporiae } \\
\text { Subhedar \& Rao ex Hosag. }\end{array}$ & Unidentified Plant sp.10 & 201099 & 17.10.2012 & $\begin{array}{l}\text { On the way to } \\
\text { Dahanu, Greater } \\
\text { Thane }\end{array}$ & 19.596222 & 72.912617 & 92 \\
\hline 151 & $\begin{array}{l}\text { Sarcinella loranthacearum } \\
\text { Hosag., Jac. Thomas \& D.K. } \\
\text { Agarwal }\end{array}$ & $\begin{array}{l}\text { Firmiana colorata (Roxb.) } \\
\text { R.Br. }\end{array}$ & 201149 & 16.10 .2012 & $\begin{array}{l}\text { Suryamal, } \\
\text { Greater Thane }\end{array}$ & 19.755400 & 73.350091 & 477 \\
\hline 152 & $\begin{array}{l}\text { Sarocladium strictum (W. Gams) } \\
\text { Summerb. }\end{array}$ & Ludwigia sp. & 196383 & 25.09 .2013 & $\begin{array}{l}\text { Sanjay Gandhi } \\
\text { National Park, } \\
\text { Greater Thane }\end{array}$ & 19.195361 & 72.906868 & 86 \\
\hline 153 & Septoria sp. & Septoria sp. & 200159 & 21.01.2012 & $\begin{array}{l}\text { Kesari, } \\
\text { Sindhudurg } \\
\text { Dist. }\end{array}$ & 15.917294 & 73.941860 & 165 \\
\hline
\end{tabular}


Table 1 Continued.

\begin{tabular}{|c|c|c|c|c|c|c|c|c|}
\hline $\begin{array}{l}\text { Sr. } \\
\text { No. }\end{array}$ & Fungi & Host plants & \begin{tabular}{|l|} 
Collection \\
No. BSI \\
(WC) \\
\end{tabular} & $\begin{array}{l}\text { Date of } \\
\text { collection }\end{array}$ & Sampling sites & $\begin{array}{l}\text { Latitude } \\
\text { (N) }\end{array}$ & $\begin{array}{l}\text { Longitude } \\
\text { (E) }\end{array}$ & $\begin{array}{l}\text { Altitude } \\
\text { (m) }\end{array}$ \\
\hline 154 & $\begin{array}{l}\text { Sheathnema indicum Dubey \& } \\
\text { Moonnambeth }\end{array}$ & $\begin{array}{l}\text { Pandanus tectorius } \\
\text { Parkinson ex Du Roi }\end{array}$ & 200140 & 21.01.2012 & $\begin{array}{l}\text { Fanaswadi, } \\
\text { Sindhudurg } \\
\text { Dist. }\end{array}$ & 15.890990 & 73.933043 & 151 \\
\hline 155 & $\begin{array}{l}\text { Solicorynespora matherensis } \\
\text { Dubey \& Moonambeth }\end{array}$ & $\begin{array}{l}\text { Roystonea regia (Kunth) } \\
\text { O.F.Cook }\end{array}$ & 196344 & 23.09 .2013 & $\begin{array}{l}\text { Matheran, } \\
\text { Raigad Dist. }\end{array}$ & 18.963105 & 73.264070 & 742 \\
\hline 156 & $\begin{array}{l}\text { Sordaria fimicola (Rob.) Ces. \& } \\
\text { de Not }\end{array}$ & $\begin{array}{l}\text { Pandanus tectorious Sol. } \\
\text { Ex Park. }\end{array}$ & 201169 & 19.10.2012 & $\begin{array}{l}\text { Tansa WLS, } \\
\text { Greater Thane }\end{array}$ & 19.529328 & 73.258630 & 295 \\
\hline \multirow[t]{4}{*}{157} & \multirow[t]{4}{*}{ Sphacelia sp. } & Diploclisia sp. & 200200 & 22.01 .2012 & $\begin{array}{l}\text { Amboli Ghat, } \\
\text { Sindhudurg } \\
\text { Dist. }\end{array}$ & 15.964716 & 74.003571 & 700 \\
\hline & & Grewia sp. & 200230 & 23.01.2012 & $\begin{array}{l}\text { Amboli Ghat, } \\
\text { Sindhudurg } \\
\text { Dist. } \\
\end{array}$ & 15.964716 & 74.003571 & 700 \\
\hline & & Ziziphus jujuba Mill & 200139 & 21.01.2012 & $\begin{array}{l}\text { Fanaswadi, } \\
\text { Sindhudurg } \\
\text { Dist. }\end{array}$ & 15.890990 & 73.933043 & 151 \\
\hline & & Unidentified plant sp. 11 & 200092 & 21.01 .2012 & $\begin{array}{l}\text { Kudal, } \\
\text { Sindhudurg } \\
\text { Dist. }\end{array}$ & 15.999956 & 73.683052 & 35 \\
\hline 158 & $\begin{array}{l}\text { Spiropes capensis (Thüm.) M.B. } \\
\text { Ellis }\end{array}$ & Vitex negundo $\mathrm{L}$. & 200081 & 21.01.2012 & $\begin{array}{l}\text { Kudal, } \\
\text { Sindhudurg } \\
\text { Dist. } \\
\end{array}$ & 15.999956 & 73.683052 & 35 \\
\hline 159 & Spiropes effusus (Pat.) M.B. Ellis & Ixora brachiata Roxb. & 200178 & 22.01 .2012 & $\begin{array}{l}\text { Amboli Ghat, } \\
\text { Sindhudurg } \\
\text { Dist. } \\
\end{array}$ & 15.964716 & 74.003571 & 700 \\
\hline \multirow[t]{2}{*}{160} & \multirow[t]{2}{*}{$\begin{array}{l}\text { Spiropes guareicola (F. Stevens) } \\
\text { Cif. }\end{array}$} & Citrus medica $\mathrm{L}$. & 200208 & 22.01 .2012 & $\begin{array}{l}\text { Amboli Ghat, } \\
\text { Sindhudurg } \\
\text { Dist. }\end{array}$ & 15.964716 & 74.003571 & 700 \\
\hline & & Ixora brachiata Roxb. & 194058 & 26.01 .2013 & $\begin{array}{l}\text { Dapoli Forest } \\
\text { Range, Ratnagiri } \\
\text { Dist. }\end{array}$ & 17.786041 & 73.152662 & 60 \\
\hline
\end{tabular}


Table 1 Continued.

\begin{tabular}{|c|c|c|c|c|c|c|c|c|}
\hline $\begin{array}{l}\text { Sr. } \\
\text { No. }\end{array}$ & Fungi & Host plants & $\begin{array}{l}\text { Collection } \\
\text { No. BSI } \\
\text { (WC) }\end{array}$ & $\begin{array}{l}\text { Date of } \\
\text { collection }\end{array}$ & Sampling sites & $\begin{array}{l}\text { Latitude } \\
\text { (N) }\end{array}$ & $\begin{array}{l}\text { Longitude } \\
\text { (E) }\end{array}$ & $\begin{array}{l}\text { Altitude } \\
\text { (m) }\end{array}$ \\
\hline & & Careya arborea Roxb. & 194063 & 26.01 .2013 & $\begin{array}{l}\text { Dapoli Forest } \\
\text { Range, Ratnagiri } \\
\text { Dist. }\end{array}$ & 17.786041 & 73.152662 & 60 \\
\hline 161 & $\begin{array}{l}\text { Spiropes japonicus (Henn.) M.B. } \\
\text { Ellis }\end{array}$ & Vites negundo L. & 200081 & 21.01 .2012 & $\begin{array}{l}\text { Kudal, } \\
\text { Sindhudurg } \\
\text { Dist. } \\
\end{array}$ & 15.999956 & 73.683052 & 35 \\
\hline \multirow[t]{2}{*}{162} & \multirow[t]{2}{*}{$\begin{array}{l}\text { Spiropes melanoplaca (Berk. \& } \\
\text { M.A. Curtis) M.B. Ellis }\end{array}$} & Mangifera indica $\mathrm{L}$. & 200141 & 21.01 .2012 & $\begin{array}{l}\text { Fanaswadi, } \\
\text { Sindhudurg } \\
\text { Dist. }\end{array}$ & 15.890990 & 73.933043 & 151 \\
\hline & & Volkameria inermis L. & 200085 & 21.01 .2012 & \begin{tabular}{|l} 
Kudal, \\
Sindhudurg \\
Dist. \\
\end{tabular} & 15.999956 & 73.683052 & 35 \\
\hline 163 & Spiropes nothofagi M.B. Ellis & Entada rheedii Spreng. & 200171 & 22.01 .2012 & $\begin{array}{l}\text { Amboli Ghat, } \\
\text { Sindhudurg } \\
\text { Dist. } \\
\end{array}$ & 15.964716 & 74.003571 & 700 \\
\hline 164 & $\begin{array}{l}\text { Stachybotrys echinata (Rivolta) } \\
\text { G. Sm. Syn. }\end{array}$ & $\begin{array}{l}\text { Roystonea regia (Kunth) } \\
\text { O.F.Cook }\end{array}$ & 196344 & 23.09 .2013 & $\begin{array}{l}\text { Matheran, } \\
\text { Raigad Dist. }\end{array}$ & 18.963105 & 73.264070 & 742 \\
\hline \multirow[t]{2}{*}{165} & \multirow[t]{2}{*}{$\begin{array}{l}\text { Stachybotrys levispora (Subram.) } \\
\text { Yong Wang bis, K.D. Hyde, } \\
\text { McKenzie, Y.L. Jiang \& D.W. Li } \\
\text { syn. }\end{array}$} & Ixora coccinea $\mathrm{L}$. & 200103 & 21.01.2012 & $\begin{array}{l}\text { Kesari, } \\
\text { Sindhudurg } \\
\text { Dist. }\end{array}$ & 15.917294 & 73.941860 & 165 \\
\hline & & Agave americana $\mathrm{L}$. & 201112 & 17.10.2012 & $\begin{array}{l}\text { On the way to } \\
\text { Dahanu, Greater } \\
\text { Thane }\end{array}$ & 19.596222 & 72.912617 & 92 \\
\hline 166 & $\begin{array}{l}\text { Stauronema sacchari Syd., P. } \\
\text { Syd. \& E.J. Butler }\end{array}$ & Coix lacryma-jobi L. & 201005 & 16.10 .2012 & $\begin{array}{l}\text { On the way to } \\
\text { Zap, Greater } \\
\text { Thane } \\
\end{array}$ & 19.898442 & 73.228724 & 375 \\
\hline 167 & $\begin{array}{l}\text { Stauronema spinificis Subhedar } \\
\text { \& V.G. Rao }\end{array}$ & $\begin{array}{l}\text { Spinifex littoreus (Burm.f.) } \\
\text { Merr. }\end{array}$ & 200238 & 22.01 .2012 & $\begin{array}{l}\text { Vengurla, } \\
\text { Sindhudurg } \\
\text { Dist. }\end{array}$ & 15.863050 & 73.642144 & 11 \\
\hline
\end{tabular}


Table 1 Continued.

\begin{tabular}{|c|c|c|c|c|c|c|c|c|}
\hline $\begin{array}{l}\text { Sr. } \\
\text { No. }\end{array}$ & Fungi & Host plants & $\begin{array}{l}\text { Collection } \\
\text { No. BSI } \\
\text { (WC) }\end{array}$ & $\begin{array}{l}\text { Date of } \\
\text { collection }\end{array}$ & Sampling sites & $\begin{array}{l}\text { Latitude } \\
(\mathbf{N})\end{array}$ & $\begin{array}{l}\text { Longitude } \\
\text { (E) }\end{array}$ & $\begin{array}{l}\text { Altitude } \\
\text { (m) }\end{array}$ \\
\hline 168 & Stenella araguata Syd. & Mesua ferrea $\mathrm{L}$. & 200120 & 21.01 .2012 & $\begin{array}{l}\text { Kesari, } \\
\text { Sindhudurg } \\
\text { Dist. }\end{array}$ & 15.917294 & 73.941860 & 165 \\
\hline 169 & Stenella plectroniae Ponnappa & Carissa congesta Wight & 200011 & 20.01.2012 & $\begin{array}{l}\text { Akeri, } \\
\text { Sawantwadi, } \\
\text { Sindhudurg } \\
\text { Dist. }\end{array}$ & 15.937989 & 73.765437 & 66 \\
\hline 170 & Temerariomyces indicum Dubey & $\begin{array}{l}\text { Nymphae rubra Roxb. ex } \\
\text { Andrews }\end{array}$ & 196372 & 24.09 .2013 & $\begin{array}{l}\text { On the way to } \\
\text { Sanjay Gandhi } \\
\text { National Park, } \\
\text { Greater Thane }\end{array}$ & 19.398960 & 72.885344 & 71 \\
\hline 171 & Tetraploa ellisii Cooke & Ixora coccinea $\mathrm{L}$. & 200103 & 21.01.2012 & $\begin{array}{l}\text { Kesari, } \\
\text { Sindhudurg } \\
\text { Dist. }\end{array}$ & 15.917294 & 73.941860 & 165 \\
\hline 172 & $\begin{array}{l}\text { Thirumalacharia thanensis } \\
\text { Dubey }\end{array}$ & Coix lacryma-jobi L. & 201125 & 18.10.2012, & $\begin{array}{l}\text { Pulachiwadi, } \\
\text { Greater Thane }\end{array}$ & 19.880359 & 73.389547 & 395 \\
\hline \multirow[t]{3}{*}{173} & \multirow[t]{3}{*}{ Torula herbarum (Pers.) Link } & Dracaena sp. & 201048 & 16.10 .2012 & $\begin{array}{l}\text { Jawhar, Greater } \\
\text { Thane }\end{array}$ & 19.917037 & 73.222610 & 433 \\
\hline & & Croton sp. & 201057 & 16.10 .2012 & $\begin{array}{l}\text { Jawhar, Greater } \\
\text { Thane }\end{array}$ & 19.917037 & 73.222610 & 433 \\
\hline & & Agave americana L. & 201112 & 17.10.2012 & $\begin{array}{l}\text { On the way to } \\
\text { Dahanu, Greater } \\
\text { Thane }\end{array}$ & 19.596222 & 72.912617 & 92 \\
\hline 174 & $\begin{array}{l}\text { Trichoconiella padwickii } \\
\text { (Ganguly) B.L. Jain }\end{array}$ & Achyranthes aspera L. & 201143 & 18.10.2012 & $\begin{array}{l}\text { Pulachiwadi, } \\
\text { Greater Thane }\end{array}$ & 19.880359 & 73.389547 & 395 \\
\hline \multirow[t]{2}{*}{175} & \multirow[t]{2}{*}{$\begin{array}{l}\text { Tripospermum acrobaticum F.B. } \\
\text { Rocha \& R.W. Barreto }\end{array}$} & $\begin{array}{l}\text { Thespesia lampas (Cav.) } \\
\text { Dalzell }\end{array}$ & 201127 & 18.10 .2012 & $\begin{array}{l}\text { Pulachiwadi, } \\
\text { Greater Thane }\end{array}$ & 19.880359 & 73.389547 & 395 \\
\hline & & $\begin{array}{l}\text { Leea indica (Burm. f.) } \\
\text { Merr. }\end{array}$ & 201152 & 18.10 .2012 & $\begin{array}{l}\text { Suryamal, } \\
\text { Greater Thane }\end{array}$ & 19.755400 & 73.350091 & 477 \\
\hline
\end{tabular}


Table 1 Continued.

\begin{tabular}{|c|c|c|c|c|c|c|c|c|}
\hline $\begin{array}{l}\text { Sr. } \\
\text { No. }\end{array}$ & Fungi & Host plants & \begin{tabular}{|l|} 
Collection \\
No. BSI \\
(WC)
\end{tabular} & $\begin{array}{l}\text { Date of } \\
\text { collection }\end{array}$ & Sampling sites & $\begin{array}{l}\text { Latitude } \\
(\mathbf{N})\end{array}$ & $\begin{array}{l}\text { Longitude } \\
\text { (E) }\end{array}$ & $\begin{array}{l}\text { Altitude } \\
\text { (m) }\end{array}$ \\
\hline \multirow[t]{4}{*}{176} & \multirow[t]{4}{*}{$\begin{array}{l}\text { Tripospermum myrti (Lind) S. } \\
\text { Hughes }\end{array}$} & Mussaenda sp. & 200044 & 20.01 .2012 & $\begin{array}{l}\text { Akeri, } \\
\text { Sawantwadi, } \\
\text { Sindhudurg } \\
\text { Dist. }\end{array}$ & 15.937989 & 73.765437 & 66 \\
\hline & & $\begin{array}{l}\text { Triumfetta rhomboidea } \\
\text { Jacq. }\end{array}$ & 201139 & 18.10 .2012 & $\begin{array}{l}\text { Pulachiwadi, } \\
\text { Greater Thane }\end{array}$ & 19.880359 & 73.389547 & 395 \\
\hline & & Combretaceae sp. 1 & 201117 & 18.10 .2012 & $\begin{array}{l}\text { Pulachiwadi, } \\
\text { Greater Thane } \\
\end{array}$ & 19.880359 & 73.389547 & 395 \\
\hline & & Dalbergia sp. & 200267 & 23.01.2012 & $\begin{array}{l}\text { Vengurla, } \\
\text { Sindhudurg } \\
\text { Dist. }\end{array}$ & 15.863050 & 73.642144 & 11 \\
\hline 177 & Vamsapriya indica Gawas \& Bhat & $\begin{array}{l}\text { Unidentified Leguminosae } \\
\text { sp. } 1\end{array}$ & 200119 & 21.01 .2012 & $\begin{array}{l}\text { Kesari, } \\
\text { Sindhudurg } \\
\text { Dist. }\end{array}$ & 15.917294 & 73.941860 & 165 \\
\hline 178 & $\begin{array}{l}\text { Veronaeopsis simplex } \\
\text { (Papendorf) Arzanlou \& Crous }\end{array}$ & Piper nigrum $\mathrm{L}$. & 200065 & 20.01 .2012 & $\begin{array}{l}\text { Sawantwadi, } \\
\text { Sindhudurg } \\
\text { Dist. }\end{array}$ & 15.904231 & 73.812647 & 184 \\
\hline 179 & Verticillium lecanii & Verticillium lecanii & 200191 & 22.01.2012 & $\begin{array}{l}\text { Amboli Ghat, } \\
\text { Sindhudurg } \\
\text { Dist. } \\
\end{array}$ & 15.964716 & 74.003571 & 700 \\
\hline \multirow[t]{2}{*}{180} & \multirow[t]{2}{*}{$\begin{array}{l}\text { Virgariella globigera (Sacc. \& } \\
\text { Ellis) S. Hughes }\end{array}$} & $\begin{array}{l}\text { Memecylon umbellatum } \\
\text { Burm. f. }\end{array}$ & 201013 & 16.10 .2012 & $\begin{array}{l}\text { On the way to } \\
\text { Zap, Greater } \\
\text { Thane }\end{array}$ & 19.898442 & 73.228724 & 375 \\
\hline & & Helictores isora $\mathrm{L}$. & 196409 & 25.09 .2013 & $\begin{array}{l}\text { Sanjay Gandhi } \\
\text { National Park, } \\
\text { Greater Thane }\end{array}$ & 19.195361 & 72.906868 & 86 \\
\hline 181 & Vizella oleariae Swart. & Ixora coccinea $\mathrm{L}$. & 200103 & 21.01.2012 & $\begin{array}{l}\text { Kesari, } \\
\text { Sindhudurg } \\
\text { Dist. }\end{array}$ & 15.917294 & 73.941860 & 165 \\
\hline 182 & Volutella sp. & Gnetum ula Brongn. & 200174 & 22.01 .2012 & $\begin{array}{l}\text { Amboli Ghat, } \\
\text { Sindhudurg } \\
\text { Dist. }\end{array}$ & 15.964716 & 74.003571 & 700 \\
\hline
\end{tabular}


Table 1 Continued.

\begin{tabular}{|c|c|c|c|c|c|c|c|c|}
\hline $\begin{array}{l}\text { Sr. } \\
\text { No. }\end{array}$ & Fungi & Host plants & $\begin{array}{l}\text { Collection } \\
\text { No. BSI } \\
\text { (WC) }\end{array}$ & $\begin{array}{l}\text { Date of } \\
\text { collection }\end{array}$ & Sampling sites & $\begin{array}{l}\text { Latitude } \\
\text { (N) }\end{array}$ & $\begin{array}{l}\text { Longitude } \\
\text { (E) }\end{array}$ & $\begin{array}{l}\text { Altitude } \\
\text { (m) }\end{array}$ \\
\hline \multirow[t]{2}{*}{183} & \multirow[t]{2}{*}{$\begin{array}{l}\text { Volutina concentrica Penz. \& } \\
\text { Sacc. }\end{array}$} & Coix lacryma-jobi L. & 201125 & 18.10 .2012 & $\begin{array}{l}\text { Pulachiwadi, } \\
\text { Greater Thane }\end{array}$ & 19.880359 & 73.389547 & 395 \\
\hline & & Areca catechu $\mathrm{L}$. & 200061 & 20.01 .2012 & $\begin{array}{l}\text { Sawantwadi, } \\
\text { Sindhudurg } \\
\text { Dist. }\end{array}$ & 15.904231 & 73.812647 & 184 \\
\hline 184 & $\begin{array}{l}\text { Wiesneriomyces laurinus (Tassi) } \\
\text { P.M. Kirk }\end{array}$ & $\begin{array}{l}\text { Pandanus tectorious Sol. } \\
\text { Ex Park. }\end{array}$ & 201169 & 19.10.2012 & $\begin{array}{l}\text { Tansa WLS, } \\
\text { Greater Thane }\end{array}$ & 19.529328 & 73.258630 & 295 \\
\hline 185 & $\begin{array}{l}\text { Xepiculopsis graminea (Lib.) Nag } \\
\text { Raj }\end{array}$ & Coix lacryma-jobi L. & 201125 & 18.10 .2012 & $\begin{array}{l}\text { Pulachiwadi, } \\
\text { Greater Thane }\end{array}$ & 19.880359 & 73.389547 & 395 \\
\hline 186 & $\begin{array}{l}\text { Zasmidium rubiacearum (S. } \\
\text { Chaudhary, N. Sharma \& Kamal) }\end{array}$ & Meyna laxiflora Robyns. & 201018 & 16.10 .2012 & $\begin{array}{l}\text { On the way to } \\
\text { Zap, Greater } \\
\text { Thane }\end{array}$ & 19.898442 & 73.228724 & 375 \\
\hline 187 & Zygosporium dilleni Dubey & Dillenia pentagyna Roxb. & 200145 & 21.01.2012 & $\begin{array}{l}\text { Fanaswadi, } \\
\text { Sindhudurg } \\
\text { Dist. }\end{array}$ & 15.890990 & 73.933043 & 151 \\
\hline \multirow[t]{5}{*}{188} & \multirow[t]{5}{*}{$\begin{array}{l}\text { Zygosporium gibbum (Sacc., M. } \\
\text { Rousseau \& E. Bommer) S. } \\
\text { Hughes }\end{array}$} & Mangifera indica $\mathrm{L}$. & 200219 & 22.01 .2012 & $\begin{array}{l}\text { Amboli Ghat, } \\
\text { Sindhudurg } \\
\text { Dist. }\end{array}$ & 15.964716 & 74.003571 & 700 \\
\hline & & Cocos nucifera $\mathrm{L}$. & 194061 & 26.01 .2013 & $\begin{array}{l}\text { Dapoli Forest } \\
\text { Range, Ratnagiri } \\
\text { Dist. }\end{array}$ & 17.786041 & 73.152662 & 60 \\
\hline & & Pogostemon sp. & 200110 & 21.01.2012 & $\begin{array}{l}\text { Kesari, } \\
\text { Sindhudurg } \\
\text { Dist. }\end{array}$ & 15.917294 & 73.941860 & 165 \\
\hline & & $\begin{array}{l}\text { Leea indica (Burm. f.) } \\
\text { Merr. }\end{array}$ & 200111 & 21.01.2012 & $\begin{array}{l}\text { Kesari, } \\
\text { Sindhudurg } \\
\text { Dist. }\end{array}$ & 15.917294 & 73.941860 & 165 \\
\hline & & $\begin{array}{l}\text { Dalbergia latifolia Roxb. } \\
\text { Ixora coccinea } \mathrm{L} \text {. }\end{array}$ & $\begin{array}{l}200998 \\
200077\end{array}$ & $\begin{array}{l}24.01 .2013 \\
21.01 .2012 \\
\end{array}$ & $\begin{array}{l}\text { Kodawali- } \\
\text { Rajapura, } \\
\text { Ratnagiri } \\
\text { Kudal, } \\
\text { Sindhudurg } \\
\end{array}$ & $\begin{array}{l}16.682105 \\
15.999956\end{array}$ & $\begin{array}{l}73.544952 \\
73.683052\end{array}$ & $\begin{array}{l}167 \\
35\end{array}$ \\
\hline
\end{tabular}


Table 1 Continued.

\begin{tabular}{|c|c|c|c|c|c|c|c|c|}
\hline $\begin{array}{l}\text { Sr. } \\
\text { No. }\end{array}$ & Fungi & Host plants & $\begin{array}{l}\text { Collection } \\
\text { No. BSI } \\
\text { (WC) }\end{array}$ & $\begin{array}{l}\text { Date of } \\
\text { collection }\end{array}$ & Sampling sites & $\begin{array}{l}\text { Latitude } \\
\text { (N) }\end{array}$ & $\begin{array}{l}\text { Longitude } \\
\text { (E) }\end{array}$ & $\begin{array}{l}\text { Altitude } \\
\text { (m) }\end{array}$ \\
\hline & & & & & Dist. & & & \\
\hline & & $\begin{array}{l}\text { Leea indica (Burm. f.) } \\
\text { Merr. }\end{array}$ & 200095 & 21.01.2012 & $\begin{array}{l}\text { Kudal, } \\
\text { Sindhudurg } \\
\text { Dist. } \\
\end{array}$ & 15.999956 & 73.683052 & 35 \\
\hline & & $\begin{array}{l}\text { Madhuca longifolia var. } \\
\text { latifolia (Roxb.) Chev }\end{array}$ & 201091 & 17.10.2012 & $\begin{array}{l}\text { On the way to } \\
\text { Dahanu, Greater } \\
\text { Thane }\end{array}$ & 19.596222 & 72.912617 & 92 \\
\hline \multirow[t]{6}{*}{189} & \multirow[t]{6}{*}{ Zygosporium masonii S. Hughes } & $\begin{array}{l}\text { Artocarpus heterophyllus } \\
\text { Lam. }\end{array}$ & 200024 & 20.01 .2012 & $\begin{array}{l}\text { Akeri, } \\
\text { Sawantwadi, } \\
\text { Sindhudurg } \\
\text { Dist. }\end{array}$ & 15.937989 & 73.765437 & 66 \\
\hline & & $\begin{array}{l}\text { Clerodendrum } \\
\text { grandiflorum (Hook.) } \\
\text { Schauer }\end{array}$ & 200214 & 22.01 .2012 & \begin{tabular}{|l} 
Amboli Ghat, \\
Sindhudurg \\
Dist. \\
\end{tabular} & 15.964716 & 74.003571 & 700 \\
\hline & & Mangifera indica $\mathrm{L}$. & 200219 & 22.01 .2012 & $\begin{array}{l}\text { Amboli Ghat, } \\
\text { Sindhudurg } \\
\text { Dist. } \\
\end{array}$ & 15.964716 & 74.003571 & 700 \\
\hline & & Ficus benghalensis $\mathrm{L}$. & 196361 & 24.09 .2013 & $\begin{array}{l}\text { Karjat, Raigad } \\
\text { Dist. } \\
\end{array}$ & 18.928594 & 73.432723 & 122 \\
\hline & & Ficus religiosa $\mathrm{L}$. & 200118 & 21.01.2012 & \begin{tabular}{|l} 
Kesari, \\
Sindhudurg \\
Dist. \\
\end{tabular} & 15.917294 & 73.941860 & 165 \\
\hline & & $\begin{array}{l}\text { Leea indica (Burm. f.) } \\
\text { Merr. }\end{array}$ & 200095 & 21.01.2012 & $\begin{array}{l}\text { Kudal, } \\
\text { Sindhudurg } \\
\text { Dist. }\end{array}$ & 15.999956 & 73.683052 & 35 \\
\hline 190 & Zygosporium minus S. Hughes & Strobilanthes callosa Nees & 201145 & 18.10 .2012 & \begin{tabular}{|l|} 
Suryamal, \\
Greater Thane
\end{tabular} & 19.755400 & 73.350091 & 477 \\
\hline 191 & Zygosporium oscheoides Mont. & $\begin{array}{l}\text { Bambusa vulgaris Schrad. } \\
\text { ex J.C.Wendl }\end{array}$ & 196378 & 24.09 .2013 & $\begin{array}{l}\text { Sanjay Gandhi } \\
\text { National Park, } \\
\text { Greater Thane }\end{array}$ & 19.195361 & 72.906868 & 86 \\
\hline
\end{tabular}


Table 2 OLS regressions

\begin{tabular}{|l|l|l|}
\hline \multirow{2}{*}{ Dependent Variable } & Model 1 & Model 2 \\
\cline { 2 - 3 } & SpsCount & In_SpsCount \\
\hline Const & $\begin{array}{l}2.2307 \\
(1.373)\end{array}$ & $\begin{array}{l}0.5513^{* * *} \\
(0.195)\end{array}$ \\
\hline DS & $\begin{array}{l}16.7692 * * * \\
(2.3208)\end{array}$ & $\begin{array}{l}2.2865^{* * *} \\
(0.3296)\end{array}$ \\
\hline DRd & $\begin{array}{l}0.7692 \\
(2.3208)\end{array}$ & $\begin{array}{l}0.1737 \\
(0.3296)\end{array}$ \\
\hline DT & $\begin{array}{l}6.1692 * * * \\
(2.0822)\end{array}$ & $\begin{array}{l}1.3815 * * * \\
(0.2957)\end{array}$ \\
\hline No. observations & 37 & 37 \\
\hline R-squared & 0.6393 & 0.6467 \\
\hline Adjusted R-squared & 0.6065 & 0.6146 \\
\hline F(3, 33) & 19.4992 & 20.1324 \\
\hline P-value(F) & $1.87 \mathrm{E}-07$ & $1.34 \mathrm{E}-07$ \\
\hline Sum squared resid & 808.7077 & 16.3137 \\
\hline S.E. of regression & 4.9504 & 0.7031 \\
\hline Log-likelihood & -109.5643 & -37.3508 \\
\hline Akaike criterion & 227.1287 & 82.7017 \\
\hline
\end{tabular}

Standard errors in parentheses.

$* \mathrm{p}<0.1, * * \mathrm{p}<0.05, * * * \mathrm{p}<.0 .01$

Table 3 Count Data Models.

\begin{tabular}{|l|l|l|}
\hline Dependent Variable & SpsCount & \multicolumn{2}{|l|}{} \\
\hline Method of Estimation & MLE & NBRM (Negbin 2) \\
\hline No. Observations & 37 & $0.8023^{* * *}$ \\
\hline Model & PRM & $(0.225)$ \\
\hline Const & $0.8023^{* * *}$ & $2.1421^{* * *}$ \\
& $(0.1857)$ & $(0.297)$ \\
\hline DS & $2.1421^{* * *}$ & 0.2963 \\
& $(0.2049)$ & $(0.3582)$ \\
\hline DRd & 0.2963 & $1.3259^{* * *}$ \\
& $(0.2865)$ & $(0.2891)$ \\
\hline DT & $1.3259 * * *$ & $0.2101 * *$ \\
& $(0.2154)$ & $(0.0913)$ \\
\hline$\phi$ & - & $2.089 \mathrm{E}-08$ \\
\hline LLR p-value & & 196.3352 \\
\hline AIC & $2.290 \mathrm{E}-38$ & - \\
\hline Chi-square test for & 215.322 & \\
Overdispersion & $0.0223^{* *}$ & \\
\hline
\end{tabular}

Standard errors in parentheses.

$* \mathrm{p}<0.1, * * \mathrm{p}<0.05, * * * \mathrm{p}<0.01$ 
Table 4 Jaccard Similarity Index (JSI)

\begin{tabular}{|l|l|l|l|l|}
\hline & $\begin{array}{l}\text { Greater } \\
\text { Thane }\end{array}$ & Raigad & Ratnagiri & Sindhudurg \\
\hline Greater Thane & 1 & & & \\
\hline Raigad & 0.03409 & 1 & & \\
\hline Ratnagiri & 0.05319 & 0 & 1 & \\
\hline Sindhudurg & 0.07927 & 0.01667 & 0.07438 & 1 \\
\hline
\end{tabular}

Species common in all the districts $=0$

Percentage of species common in maximum number of districts (i.e. Greater Thane, Ratnagiri, Sindhudurg) $=4.396 \%$

\section{Discussion}

In present study, a total of 191 foliicolous micro-fungi were found from Konkan region. There was highly statistically significant difference in mean species count, thereby biodiversity, across the districts $\left(\mathrm{F}_{\mathrm{p} \text {-value }}<0.01\right)$. OLS (Model 1) and Count Data models initially showed residuals not having normal distribution, however by dropping the largest observation (SpsCount $=$ 33 for Kesari), modified models showed normally distributed residuals with similar results at same level of statistical significance $(\alpha=0.01$ ), hence original models were retained. Model 1 , both original and modified (by dropping Kesari observation), showed highly significant (p-value $<0.01$ ) heteroskedasticity for both the tests. Hence Model 1 was modified by $\log$ transformation of the dependent variable (Model 2). Model 2 showed results similar to Model 1, with no heteroskedasticity and normally distributed residuals, proving in this case that non-normal and heteroskedastic residuals did not affect overall significance of regression and significance of estimated coefficients of Model 1. Also, despite significant overdispersion ( $p$-value < 0.05), PRM yielded results similar to NBRM at same level of statistical significance $(\alpha=0.01)$. Count data models are generally recommended for large samples. However, in present study, in terms of overall significance of regression and significance of estimated individual coefficients, they show results similar to OLS models. Konkan also showed very high dissimilarity in species composition across the districts, with highest JSI being $7.927 \%$, none of the species being common to all district and only 4 species ( $4.396 \%$ of total) being common in maximum 3 districts. In future, the same framework can be adopted in other similar studies. The statistical analysis carried out is based on the data obtained from the study. However, it should be noted that the statistical results can't be generalized as diverse ecological features and sheer number of fungi (many yet to be discovered) can yield different results.

\section{Acknowledgement}

Authors are thankful to the Director, Botanical survey of India, for his kind support and providing all the research facilities. They are also grateful to the Head of the office, Botanical Survey of India, Western Regional Centre, Pune, for his kind support and encouragement. Ministry of Environment, Forest \& Climate Change, New Delhi, and Forest Department, Government of Maharashtra are thankfully acknowledged.

\section{References}

Ainsworth GC. 1973 - Introduction and keys to higher taxa. In: The fungi, vol. IV. Eds., G. C. Ainsworth, F. K. Sparrow, and A. S. Sussman. Academic Press, New York, pp. 1-7.

Alexopolous CJ, Mims CW. 1979 - Introductory Mycology, 3rd ed., John Wiley and Sons, New York.

Andrews JH, Harris RF. 2000 - The ecology and biogeography of microorganisms on plant surfaces. Annual Review of Phytopathology 38, 145-180. 
Andrews JH, Hirano SS. 1991 - Microbial ecology of leaves. Brock/Springer series in contemporary bioscience (USA).

Von Arx JA. 1981 - The genera of fungi sporulating in pure culture (No. Ed. 3). J. Cramer.

Barnett HL, Hunter BB. 1972 - Illustrated genera of Imperfect Fungi. Burgess Publishing Company.

Bhat DJ, Kendrick WB. 1993 - Twenty-five new conidial fungi from the Western Ghats and the Andaman Islands (India). Mycotaxon 49, 19-90.

Bhat DJ, Raghukumar S. 2000 - Ecology of fungi. Goa University, India.

Bhat DJ. 2010 - Fascinating microfungi (Hyphomycetes) of Western Ghats, India. Broadway Book Centre, Panaji, India.

Booth C. 1971 - The genus Fusarium. CMI, Kew, Surrey, England.

Butler EJ, Bisby GR. 1931 - Fungi in India. Science Monograph. Indian Council of Agricultural Research, New Delhi.

Cottrell A, Lucchetti R. 2018 - Gretl User Manual, Available at: http://gretl.sourceforge.net/gretlhelp/gretl-guide.pdf. [Accessed 25 October 2018].

Cummins GB, Hiratsuka Y. 1983 - Illustrated Genera of Rust Fungi. Rev. ed. American Phytopathological Society Press, St. Paul, Minnesota.

Dennis RWG. 1978 - British Ascomycetes. J. Cramer.

D’Souza MA. 2002 - Studies on diversity, ecology and biology of microfungi associated with a few dicot and monocot plant species of Western Ghats in Goa State, India, PhD thesis, Goa University, India.

Ellis MB. 1971 - Dematiaceous Hyphomycetes. C.A.B. Common Wealth Mycological Institute, Kew Surrey, England.

Ellis MB. 1976 - More Dematiaceous Hyphomycetes. Commonwealth Mycological Institute, Kew Surrey, England.

Ellis MB, Ellis JP. 1985 - Micro fungi of land plants. An Identification Handbook. Biddles Ltd., Guildford \& King's Lynn.

Greene WH. 2011 - Econometric analysis, $7^{\text {th }}$ ed. Pearson Education Limited.

Gujarati D. 2014 - Econometrics by example. Macmillan International Higher Education.

Hosagoudar VB. 1996 - Meliolales of India. Botanical Survey of India, Calcutta.

Hosagoudar VB. 2008 - Meliolales of India. Vol. II. Botanical Survey of India, Calcutta.

Hosagoudar VB, Robin PJ. 2011 - Five new black mildews from the Western Ghats of Peninsular India. Bioscience Discovery Journal 2, 264-268.

Hosagoudar VB. 2012 - Asterinales of India. Mycosphere 2(5), 617-852.

Hosagoudar VB. 2013 - Meliolales of India - Volume III. Journal of Threatened Taxa 5(6), 39934068.

Kamal BR, Singh RP. 1980 - Fungi of Gorakhpur-XIX. Pseudocercospora. Sydowia 33, 157-161.

Kamal BR, Morgan-Jones G. 1985 - Notes on Hyphomycetes. L. Two new species of Sirosporium from India. Mycotaxon 24, 313-318.

Kinkel LL. 1997 - Microbial population dynamics on leaves. Annual Review of Phytopathology $35,327-347$

Karve IK. 1968 - Maharashtra, Land and Its People. Directorate of Government Printing, Stationery and Publications, Maharashtra State.

Kumar P, Kamal BR. 1979 - A new species of Pseudocercospora. Current Science 48, 128- 129.

Mall TP, Singh DP, Kumar A, Sahani S. 2013 - Foliicolus fungi: earth's living treasure from North-Tarai forests of (Uttar Pradesh) India. Indian Journal of Science 3, 88-96.

Mundkar BB. 1938 - Fungi of India (Supplement-1). ICAR Science Monograph 12, 54.

Nag Raj TR. 1993 - Coelomycetous anamorphs with appendage-bearing conidia. Mycologue publications.

Pande A, Bansude GM. 1980 - Leptosphaeria leaf-spot of Agave - a new record from India. Maharashtra Vigyan Mandir Patrika 1, 31-32. 
Pande A. 1981 - Three foliicolous fungi from India. Maharashtra Vigyan Mandir Patrika 16, 3336.

Parandekar SA. 1964 - A contribution to the fungi of Maharashtra. Journal of University of Poona, Science \& Technology Sections 26, 57-65.

Patil SD, Magdum DK. 1979 - Some Cercospora species from Ganeshkhind area. Maharashtra Vigyan Mandir Patrika 14, 49-51.

Patil MS, Pawar AB. 1989 - Studies in foliicolous fungi -V. Indian Phytopathology 42, 247-252.

Patwardhan PG. 1969 - Studies in the Powdery Mildews (Erysiphaceae) of Maharashtra (India): I ascigerous. J. Shivaji University 2, 55-57.

Pratibha J, Raghukumar S, Bhat DJ. 2010 - New species of Dendryphiopsis and Stauriella from Goa, India. Mycotaxon 113, 297-313.

Rai AA, Kamal. 1982 - A new species of Pseudocercospora Speg. Current Science 51, 287-288.

Rajak RC, Pandey AK. 1984 - A new hyphomycetes. Phaeoisariopsis chonemorphae sp. nov. Current Science 53, 150-151.

Sahni VP. 1964 - Some foliicolous ectoparasites and associated fungi from Jabalpur. Mycopathologia et Mycologia Applicata 23, 328-338.

Sawant RJ, Papdiwal PB. 2007 - Studies on leaf spot diseases of Annona squamosa L. in Beed district of Maharashtra. Bioinfolet 4, 227-228.

Seabold S, Perktold J. 2010 - Statsmodels: Econometric and statistical modeling with Python. Proceedings of the 9th Python in Science Conference.

Seifert K, Morgan-Jones G, Gams W, Kendrick B. 2011 - The Genera of Hyphomycetes. CBS Biodiversity series. 9.

Singh N, Todawat J, Papdiwal PB. 2011 - Leaf spot diseases of some fruit trees of Aurangabad district, Maharashtra. Bioinfolet 8, 87-90.

Singh NP, Karthikeyan S. 2000 - Flora of Maharashtra State, Dicotyledonae Vol 1: Ranunculaceae-Rhizophoraceae. Botanical Survey of India, Calcutta.

Sivanesan A. 1983 - The bitunicate ascomycetes and their anamorphs. Lubrecht \& Cramer Ltd.

Smith D, Onions AHS. 1994 - Preservation and Maintenance of living fungi. IMI Technical Handbook 2. CAB International, U. K.

Subramanian CV. 1971 - Hyphomycetes, an account of Indian species except Cercospora. ICAR, New Delhi.

Sutton BC. 1980 - The Coelomycetes, Fungi Imperfecti with pycnidia, acervuli andstromata. C.A.B. International Mycological Research Institute, Kew, Survey, England.

Thimmaiah CJ, Hosagoudar VB, Jayashankar M. 2013 - Black mildews of Kodagu, Karnataka. Journal of Threatened Taxa 5, 5021-5180.

Verma RK, Kamal. 1987a - Agarwalomyces indicus gen. et sp. nov. a fruticolous synematous hyphomycetes from Uttar Pradesh. Transactions of the British Mycological Society 89, 596599.

Verma RK, Kamal. 1987b - New species of Pseudocercospora, Phaeoisariopsis, Sarcinella and Stenella from Uttar Pradesh India. Transactions of the British Mycological Society 89, 67 72.

Verma RK, Kamal. 1991 - Some new species and new combinations in Pseudocercospora from India. Indian Phytopathology 44, 440 - 447.

\section{Supplementary material}

\section{Collection locations with species \\ Greater Thane}

On the way to Zap (13)

Acremoniula sarcinellae, Capnodium sp.1,

Circinotrichum olivaceum, Cladosporium

cladosporioides, Colletotrichum

gleosporioides, Colletotrichum 


\begin{tabular}{|c|c|}
\hline & $\begin{array}{l}\text { lindemuthianum, Epicoccum nigrum, Gonatophragmium mayteni, Meliola } \\
\text { carissae, Pithomyces ellisii, Stauronema sacchari, Virgariella globigera, } \\
\text { Zasmidium rubiacearum }\end{array}$ \\
\hline Shirpur(7) & $\begin{array}{l}\text { Cercospora apii, Cercospora blumeicola, Leptoxyphium glochidion, Mitteriella } \\
\text { ziziphina, Myrothecium roridum, Neopestalotiopsis asiatica, Pseudocercospora } \\
\text { eupatorii-formosanae }\end{array}$ \\
\hline Jawhar(5) & $\begin{array}{l}\text { Ampelomyces quisqualis, Asperisporium pongamiae, Chloridium indicum, } \\
\text { Phialophora cyclaminis, Torula herbarum }\end{array}$ \\
\hline $\begin{array}{l}\text { On the way to } \\
\text { Dahanu(14) }\end{array}$ & $\begin{array}{l}\text { Asterina woodfordiae, Cercospora apii, Cercospora ricinella, Cladosporium } \\
\text { gallicola, Graphium sp., Khuskia oryzae, Nigrospora sphaerica, Passalora } \\
\text { desmanthi, Pleurocytospora vestita, Sarcinella cassiae-fistulae, Sarcinella } \\
\text { gymnosporiae, Stachybotrys levispora, Torula herbarum, Zygosporium gibbum }\end{array}$ \\
\hline $\begin{array}{l}\text { Pulachiwadi } \\
\text { (18) }\end{array}$ & $\begin{array}{l}\text { Amerosporium polynematoides, Ampelomyces quisqualis, Cercospora apii, } \\
\text { Colletotrichum dematium, Colletotrichum gleosporioides, Colletotrichum } \\
\text { lindemuthianum, Corynespora torulosa, Deightoniella jabalpurensis, Drechslera } \\
\text { papendorfii, Nigrospora sphaerica, Periconia minutissima, Sarcinella gmelinae, } \\
\text { Thirumalacharia thanensis, Trichoconiella padwickii, Tripospermum } \\
\text { acrobaticum, Tripospermum myrti, Volutina concentrica, Xepiculopsis graminea }\end{array}$ \\
\hline Suryamal (3) & Sarcinella loranthacearum, Tripospermum acrobaticum, Zygosporium minus \\
\hline $\begin{array}{l}\text { Tansa WLS } \\
\text { (6) }\end{array}$ & $\begin{array}{l}\text { Ampelomyces quisqualis, Cercospora apii, Domingoella asternarum, } \\
\text { Pyriculariopsis sp., Sordaria fimicola, Wiesneriomyces laurinus }\end{array}$ \\
\hline $\begin{array}{l}\text { On the way to } \\
\text { Sanjay } \\
\text { Gandhi } \\
\text { National Park } \\
\text { (6) }\end{array}$ & $\begin{array}{l}\text { Alternaria chlamydospora, Cercospora apii, Colletotrichum dematium, } \\
\text { Fusicoccum sp., Phoma multirostrata, Temerariomyces indicum }\end{array}$ \\
\hline $\begin{array}{l}\text { Sanjay } \\
\text { Gandhi } \\
\text { National Park } \\
(10)\end{array}$ & $\begin{array}{l}\text { Chalara siamense, Helicomina costi, Meliola buteae, Meliola holarrhenae, } \\
\text { Meliola hyptidis, Meliola mitragynae, Periconia lateralis, Sarocladium strictum, } \\
\text { Virgariella globigera, Zygosporium oscheoides }\end{array}$ \\
\hline Wada (2) & Beltrania querna, Moorella speciosa \\
\hline \multicolumn{2}{|c|}{ Raigad District } \\
\hline Matheran (10) & $\begin{array}{l}\text { Ardhachandra cristaspora, Cirsosia vateriae, Coniothyrium palmarum, } \\
\text { Dictyosporium subramanianii, Fusarium solani, Microsphaeropsis sarcinellae, } \\
\text { Phaeoisaria clematidis, Pithomyces pavgii, Solicorynespora matheransis, } \\
\text { Stachybotrys echinata }\end{array}$ \\
\hline Karjat (4) & $\begin{array}{l}\text { Ardhachandra cristaspora, Colletotrichum dematium, Corynespora cassicola, } \\
\text { Zygosporium masonii }\end{array}$ \\
\hline $\begin{array}{l}\text { On the way to } \\
\text { Panvel (1) }\end{array}$ & Drechslera papendorfii \\
\hline $\begin{array}{l}\text { Phansad WLS } \\
(2)\end{array}$ & Capnodium sp.1, Meliola unonicola \\
\hline $\begin{array}{l}\text { On the way to } \\
\text { Matheran (2) }\end{array}$ & Corynespora cassicola, Echidnodella polyalthiae \\
\hline Khopoli (1) & Corynespora cassicola \\
\hline $\begin{array}{l}\text { Raigad Forest } \\
\text { (1) }\end{array}$ & Periconia cambrensis \\
\hline \multicolumn{2}{|c|}{ Ratnagiri District } \\
\hline Dapoli (2) & Bahusandhika indica, Excipulariopsis narsapurensis \\
\hline Dapoli Forest & Asterina wrightii, Domingoella asternarum, Fusarium oxysporum, Isthmospora \\
\hline
\end{tabular}




\begin{tabular}{|c|c|}
\hline Range (6) & spinosa, Spiropes guareicola, Zygosporium gibbum \\
\hline $\begin{array}{l}\text { Ganpatipule } \\
\text { (1) }\end{array}$ & Periconia cookei \\
\hline Khed (1) & Harpographium sp. \\
\hline Kirbet (2) & Meliola melanoxylonis, Repetophragma ellisii \\
\hline $\begin{array}{l}\text { Kodawali- } \\
\text { Rajapura (6) }\end{array}$ & $\begin{array}{l}\text { Acrodictys balladynae, Balladyna vanderystii, Colletotrichum lindemuthianum, } \\
\text { Phyllachora sp., Prathigada terminaliae, Zygosporium gibbum }\end{array}$ \\
\hline $\begin{array}{l}\text { Kurne-Lanja } \\
\text { Taluka } \\
\text { Reserved } \\
\text { Forest (3) }\end{array}$ & Domingoella asternarum, Goosiomyces bambusicola, Meliola bauhiniicola \\
\hline $\begin{array}{l}\text { Near } \\
\text { AmbaGhat(1) }\end{array}$ & Cladosporium colocasiae \\
\hline $\begin{array}{l}\text { On the way to } \\
\text { Khed (3) }\end{array}$ & Colletotrichum gleosporioides, Puccinia kraussiana, Rhinocladium sp. \\
\hline Pachavali (1) & Isthmospora spinosa \\
\hline Panval(1) & Custingophora ratnagiriensis \\
\hline Rajapura (1) & Penicillium notatum \\
\hline Ratnagiri (1) & Khuskia oryzae \\
\hline \multicolumn{2}{|c|}{ Sindhudurg District } \\
\hline Akeri (23) & $\begin{array}{l}\text { Aphanofalx sp., Asperisporium pongamiae, Asterina jasminicola, Balladyna } \\
\text { pavattae, Cercosporella thunbergiae, Colletotrichum gleosporioides, } \\
\text { Corynespora cassicola, Didymella fabae, Gibberella baccata, Hansfordiellopsis } \\
\text { lichenicola, Meliola careyae, Meliola desmodii-triquetri, Meliola tylophorae, } \\
\text { Neopestalotiopsis asiatica, Periconia byssoides, Pestalotiopsis funerea, } \\
\text { Porrectotheca sp., Prillieuxina polyalthiae, Pseudocercospora conyzae, } \\
\text { Pseudocercospora sp. 1, Stenella plectroniae, Tripospermum myrti, Zygosporium } \\
\text { masonii }\end{array}$ \\
\hline $\begin{array}{l}\text { AmboliGhat } \\
(24)\end{array}$ & $\begin{array}{l}\text { Aschersonia aleyrodis, Asterina capparis, Chloridium indicum, Cladosporium } \\
\text { cladosporioides, Cladosporium colocasiae, Cladosporium oxysporum, } \\
\text { Colletotrichum gleosporioides, Corynespora cassicola, Custingophora olivacea, } \\
\text { Helicoceras celtidis, Meliola eugeniae-stocksii, Meliola ixorae Yates var. } \\
\text { macrospora, Meliola ixorae-coccineae, Meliola mangiferae, Pestalotiopsis } \\
\text { linearis, Rhinocladium sp., Sphacelia sp., Spiropes effusus, Spiropes guareicola, } \\
\text { Spiropes nothofagi, Verticillium lecanii, Volutella sp., Zygosporium gibbum, } \\
\text { Zygosporium masonii }\end{array}$ \\
\hline $\begin{array}{l}\text { Fanaswadi } \\
\text { (19) }\end{array}$ & $\begin{array}{l}\text { Acarophialophora sp., Aschersonia aleyrodis, Corynespora cassicola, } \\
\text { Dendryphiella vinosa, Dendryphion state of Pleospora papaveracea, } \\
\text { Domingoella asternarum, Isthomospora state of Trichothyrium asterophorum, } \\
\text { Meliola allophyli-serrulati, Meliola eugeniae-stocksii, Meliola pandanacearum, } \\
\text { Meliola ziziphi, Pestalotiopsis anacardii, Pestalotiopsis linearis, Philonectria } \\
\text { sp., Polytretophora calcarata, Sheathnema indicum, Sphacelia sp., Spiropes } \\
\text { melanoplaca, Zygosporium dilleni }\end{array}$ \\
\hline Kesari (33) & $\begin{array}{l}\text { Amazonia elaeocarpi, Aschersonia aleyrodis, Asterina jambolanae, } \\
\text { Cladosporium colocasiae, Corynespora cassicola, Didymella fabae, Gliocladium } \\
\text { penicilloides, Glomerella cingulata, Helicosporium lumbricoides, Isthmospora } \\
\text { spinosa, Khuskia oryzae, Kirschsteiniothelia atra, Meliola alstoniae, Meliola } \\
\text { hemidesmicola, Meliola melanoxylonis, Meliola pongamiae, Meliola semecarpi- } \\
\text { anacardii, Meliola tylophorae-indicae, Meliolina mollis, Metulocladosporiella }\end{array}$ \\
\hline
\end{tabular}




\begin{tabular}{|l|l|}
\hline & $\begin{array}{l}\text { musae, Periconia lateralis, Periconiella telopeae, Pestalotiopsis linearis, Phoma } \\
\text { tropica, Questeriella strychni, Septoria sp., Stachybotrys levispora, Stenella } \\
\text { araguata, Tetraploa ellisii, Vamsapriya indica, Vizella oleariae, Zygosporium } \\
\text { gibbum, Zygosporium masonii }\end{array}$ \\
\hline Kudal(17) & $\begin{array}{l}\text { Asteridiella depokensis, Asterina jasminicola, Domingoella asternarum, } \\
\text { Isthmospora spinosa, Kirschsteiniothelia atra, Meliola eugeniae-jamboloidis, } \\
\text { Meliola hyptidis, Meliola ixorae Yates var. macrospora, Meliola jasminicola, }, \\
\text { Meliolina mollis, Pirozynskiella solaninum, Sphacelia sp., Spiropes capensis, } \\
\text { Spiropes japonicus, Spiropes melanoplaca, Zygosporium gibbum, Zygosporium } \\
\text { masonii }\end{array}$ \\
\hline Sawantwadi \\
$(9)$ & $\begin{array}{l}\text { Acroconidiellina arecae, Asterina delicatula, Cercospora careyae, } \\
\text { Cladosporium oxysporum, Mycovellosiella solani-torvi, Phoma nebulosa, } \\
\text { Puccinia phragmitis, Veronaeopsis simple, Volutina concentrica }\end{array}$ \\
\hline Vengurla(8) & $\begin{array}{l}\text { Cladosporium spongiosum, Diplodia } \text { sp., Melanocarpus sp., Mycovellosiella } \\
\text { solani-torvi, Prathigada } \text { sp., Puccinia phragmitis, Stauronema spinificis, } \\
\text { Tripospermum myrti }\end{array}$ \\
\hline
\end{tabular}

